\title{
INFINITESIMALLY RIGID POLYHEDRA. I. STATICS OF FRAMEWORKS
}

\author{
BY \\ WALTER WHITELEY ${ }^{1}$
}

\begin{abstract}
From the time of Cauchy, mathematicians have studied the motions of convex polyhedra, with the faces held rigid while changes are allowed in the dihedral angles. In the 1940s Alexandrov proved that, even with additional vertices along the natural edges, and with an arbitrary triangulation of the natural faces on these vertices, such polyhedra are infinitesimally rigid. In this paper the dual (and equivalent) concept of static rigidity for frameworks is used to describe the behavior of bar and joint frameworks built around convex (and other) polyhedra. The static techniques introduced provide a new simplified proof of Alexandrov's theorem, as well as an essential extension which characterizes the static properties of frameworks built with more general patterns on the faces, including frameworks with vertices interior to the faces.

The static techniques are presented and employed in a pattern appropriate to the extension of an arbitrary statically rigid framework built around any polyhedron (nonconvex, toroidal, etc.). The techniques are also applied to derive the static rigidity of tensegrity frameworks (with cables and struts in place of bars), and the static rigidity of frameworks projectively equivalent to known polyhedral frameworks. Finally, as an exercise to give an additional perspective to the results in 3-space, detailed analogues of Alexandrov's theorem are presented for convex 4-polytopes built as bar and joint frameworks in 4-space.
\end{abstract}

1. Introduction. Over the last decade there has been an upsurge in activity by mathematicians and engineers on problems concerning the rigidity of various structures [2-4, 7, 9-11, 14, 15, 17, 19].

This work has drawn on two distinct traditions: the engineering study of the statics of bar and joint frameworks $[16,18]$, and the mathematical study of the motions of triangulated convex polyhedra-often viewed as structures formed with rigid faces and hinges $[1,6,8,9,15,21]$. The more recent work has involved a gradual cross-over of topics, with mathematicians studying the motions of frameworks $[2,3,10,14]$ and moving on to use the static techniques $[7,20]$, while engineers resumed their interest in structures formed of rigid panels [4].

In [11] we presented a program for a unified projective geometric theory for both the statics of bar and joint frameworks and the instantaneous mechanics of hinged panel structures. The second paper [23] presented an application of the program to

\footnotetext{
Received by the editors June 2, 1982 and, in revised form, October 20, 1983.

1980 Mathematics Subject Classification. Primary 51M20, 52A25, 70C99; Secondary 73K99, 70 B99.

${ }^{1}$ Preparation of this manuscript was assisted, in part, by N.S.E.R.C. of Canada Grant A3568, and final preparation occurred during a visiting semester at Cornell University.
} 
bar and joint frameworks in the plane. In this paper we continue this program with applications to the static behavior of frameworks built around polyhedra. In a fourth paper [27] we will continue this program by applying these static techniques and results to describe the behavior of panel structures built around oriented polyhedra.

While more details about the background of this program were presented in [11], our approach here, as well as the pattern of results obtained, can be summarized as follows.

(a) Bar and joint frameworks on the surface of a polyhedron are best understood using statics (\$2). We characterize static rigidity (the ability to resolve all reasonable external loads by a response of tensions and compressions in the bars) by the size of a vector space of loads generated by the bars of the framework (Theorem 2.1 ). With this characterization we provide simple principles for modifying a framework by the addition of new joints with appropriate bars, and by the replacement of one subframework by a new subframework on the same joints-with the modifications preserving static rigidity.

(b) A triangulated sphere has $E=3 v-6$ bars on $v$ joints, and this is the precise size of the vector space of loads to be spanned. We use some techniques originating with Cauchy, and the techniques of static replacement to give a new proof that the bars of a convex sphere, with triangulated faces, are independent-and thus that this framework is statically rigid (Theorem 3.1). This result is equivalent to a theorem of Alexandrov [1, 3], but we find this proof simpler, and more intuitive.

(c) The techniques of $\$ 2$ can be used to extend any result like Theorem 3.1 to give the static rigidity of larger frameworks with additional joints on the natural edges, and with any plane-statically rigid frameworks on these joints substituted for the faces (Theorem 4.1). These replacements are explored in detail to obtain conditions for minimal statically rigid frameworks, including a particular extension of Theorem 2.1, also due to Alexandrov [1, 3].

(d) The static techniques provide an easy extension of all results obtained to tensegrity frameworks - frameworks with cables (tension members) and struts (compression members) in place of some, or all bars (\$5).

(e) The static techniques also provide a description for the behavior of the frameworks of $\S 4$ when they are extended by additional joints inside the faces $(\S 6)$. These new results can be employed to obtain results on the rigidity of panel structures [27], and to extend results on 2nd order rigidity [10].

(f) Although results through $\$ 6$ are presented in Euclidean form, the basic properties of static rigidity are projectively invariant. In $\S 7$ we translate the previous results, through projective transformations, to study the static rigidity of certain nonconvex polyhedra. We also re-examine the effects of the static substitution techniques, both before and after such projections.

(g) The theorems about frameworks in 3-space built around polyhedra have natural extensions to frameworks built around 4-polytopes in 4-space. We present basic analogues of Alexandrov's theorems for convex 4-polytopes (Theorem 8.1 and Corollary 8.3).

(h) This analysis of frameworks by static substitutions can be exploited to study a number of related problems. Some directions this work has taken are outlined in $\$ 9$. 
We first undertook this research into polyhedral frameworks under the stimulation of a series of conjectures offered by Grünbaum [14], and some of them are answered here. Gluck's presentation of the theorem of Cauchy and Dehn then provided our initial introduction to the older theory [15]. After completing a first draft of this paper, we discovered portions of $\S \S 3$ and 4 had been obtained by Alexandrov [1], using more complicated combinatorial arguments in place of the statics.

We wish to thank Ben Roth for generously sharing his rewritten form of our original proofs. His approach has clarified our perception of the essential underlying patterns of $\S \S 3-5$. We also appreciate his encouragement to present this approach, as an alternative to the approach of Alexandrov which he and Len Asimow had laboured to present English [3].

Less obvious, but more critical, is our enormous debt to the Structural Topology Research Group at l'Université de Montréal, with whom we learned about the problems of rigidity, the underlying projective patterns, and the static approach to bar and joint frameworks [5, 11]. In particular, we thank Janos Baracs, Henry Crapo and Rachad Antonius for numerous conversations and insights on the topics covered here.

We also thank the referee for this helpful and careful editorial comments.

Finally, we acknowledge the inadvertent support of the Government of Quebec which provided the leisure to pursue the original research while participating in the Quebec teachers' strike of 1976.

2. Statics of bar and joint frameworks. We begin with the promised introduction to the static rigidity of bar and joint frameworks. The equivalence of this concept to the idea of infinitesimal rigidity will be described at the close of this section.

Since we will work in various dimensions-from the line to 4-space-we offer the definitions in a $d$-dimensional form. A more leisurely (but projective) introduction to these ideas appears in [11, $\$ 2$ and 26].

Definition 2.1. A bar and joint framework in $d$-space is a set $V=\left\langle a_{1}, \ldots, a_{v}\right\rangle$ of points in $\mathbf{R}^{d}$ (the joints), and a set $B$ of unordered pairs of indices $\{i, j\}, 1 \leqslant i, j \leqslant v$ (the bars), such that if $\{i, j\} \in B$ then $a_{i} \neq a_{j}$.

The intuititive test for static rigidity of a framework is to apply external loads - forces at the joints-and investigate the response, by tensions and compressions in the bars, which gives an equilibrium at the joints. Of course, only certain loads - the equilibrium loads - can be resolved in this way. These equilibrium loads are the loads which do not correspond to any rigid motion of space.

Definition 2.2. An equilibrium load on a bar and joint framework in $d$-space is a set of $d$-vectors $\left\langle F_{1}, \ldots, F_{v}\right\rangle$ (one force for each joint), such that $\sum F_{i}=0$ (sum over all joints) and for each pair of coordinate directions $1 \leqslant h<k \leqslant d$, writing the $k$ th component as of a vector $X$ as $X_{k}$ we have

$$
\left.\sum\left(\left(F_{i}\right)_{k}\left(a_{i}\right)_{h}-\left(F_{i}\right)_{h}\left(a_{i}\right)_{k}\right)=0 \quad \text { (sum over all joints } a_{i}\right) .
$$

For a fixed set of joints the equilibrium loads form a vector space in $\mathbf{R}^{d v}$ defined by this set of $d(d+1) / 2$ linear equations. As we shall see shortly, when there are at 
least $d$ joints these equations are independent, and this vector space has dimension $d v-d(d+1) / 2$.

Definition 2.3. A resolution by a bar and joint framework of an equilibrium load is a set of scalars $\lambda_{i j}$, one for each bar, such that at each joint $a_{i}$

$$
\sum \lambda_{i j}\left(a_{i}-a_{j}\right)+F_{i}=0 \quad(\text { sum over } j \text { with }\{i, j\} \in B) .
$$

A bar and joint framework is statically rigid in $d$-space iff there is a resolution for every equilibrium load on its joints.

It is possible to embed $d$-space in a higher dimensional space, and inquire about the rigidity of a framework in this larger space. On the other hand, if the joints of a framework span (in an affine sense) only a $k$-dimensional subspace of $d$-space, then it is simple to pull this subspace out, and to speak of the $k$-dimensional static rigidity of the framework. We will do both processes freely.

For each pair of joints $a_{s}, a_{t}$, there is a simple equilibrium load with forces only at these joints

$$
F_{s t}=\left\langle 0, \ldots, 0, a_{s}-a_{t}, 0, \ldots, 0, a_{t}-a_{s}, 0, \ldots, 0\right\rangle .
$$

(It is easy to confirm that this is, indeed, an equilibrium load.) Clearly a bar $\{i, j\}$ will resolve the load $F_{i j}$. If the framework is statically rigid, then $F_{s t}$ is resolved for every pair of joints. Barring degeneracy, the converse is also true-the loads $F_{s t}$ generate the entire space of equilibrium loads, and form a test for the static rigidity of the framework.

THEOREM 2.1. A bar and joint framework is statically rigid in d-space iff (i) for all pairs of joints the load $F_{s t}$ is resolved and (ii) either the joints span d-space (in an affine sense) or there are exactly $k+1$ joints and these span a $k$-dimensional subspace.

Proof. If the framework is statically rigid, then it clearly will resolve all the $F_{s t}$. Suppose then that it does not span $d$-space and that all its $m$ joints lie in an affine space of dimension $<m-1$. Then there is an affine dependence of the joints

$$
\alpha_{1} a_{1}+\cdots+\alpha_{m} a_{m}=0 \text { and } \sum_{i} \alpha_{i}=0 .
$$

We now take a point $p$ not in this subspace and generate the external load:

$$
\left\langle\alpha_{1}\left(a_{1}-p\right), \alpha_{2}\left(a_{2}-p\right), \ldots, \alpha_{m}\left(a_{m}-p\right)\right\rangle \text {. }
$$

This is an equilibrium load, since

$$
\sum_{i} \alpha_{i}\left(a_{i}-p\right)=\sum_{i} \alpha_{i} a_{i}-\left(\sum_{i} \alpha_{i}\right) p=0
$$

and

$$
\begin{aligned}
& \sum_{i}\left(\alpha_{i}\left(a_{i}-p\right)\right)_{h}\left(a_{i}\right)_{k}-\left(\alpha_{i}\left(a_{i}-p\right)_{k}\left(a_{i}\right)_{h}\right) \\
& =\sum_{i}\left(\alpha_{i}(p)_{k}\left(a_{i}\right)_{h}-\alpha_{i}(p)_{h}\left(a_{k}\right)_{k}\right) \\
& =p_{k}\left(\sum_{i} \alpha_{i}\left(a_{i}\right)_{h}\right)-(p)_{h}\left(\sum_{i} \alpha_{i}\left(a_{i}\right)_{k}\right)=0 .
\end{aligned}
$$

Clearly this load cannot be resolved, which gives a contradiction. 
We conclude that the two conditions in the theorem are necessary for static rigidity.

We will now prove, by induction, that (i) if $k$ joints of the framework span a $(k-1)$-dimensional affine space, and all $F_{i j}$ among these $k$ joints are resolved, then the framework resolves all equilibrium loads assigned to these joints in any dimensional space $d \geqslant k-1$ and (ii) if the joints of the framework span a $d$-dimensional space and all $F_{i j}$ are resolved, then the framework is statically rigid in dimension $d$.

Dimension 1. In dimension 1, (i) is irrelevant. To prove (ii) we do an induction on the number of joints, $v$, at which the load to be resolved is applied. If $v=1$, the load is applied at a single joint. The only equilibrium force on one joint is $F=0$, a load which is resolved by the zero assignment (or the empty assignment if $B=\varnothing$ ).

Assume that we have proven that any load applied to $v$ joints of a line framework is resolved, and consider a load $F$ applied to $v+1$ joints on the line $a_{0} a_{1} a_{2} \cdots a_{v}$. We know that the load $F_{01}$ has a resolution by scalars $\beta_{i j}$, and that we can find a scalar $\alpha$ such that $F_{1}=\alpha\left(a_{0}-a_{1}\right)$. Then the load $F-\alpha F_{01}$ is applied to $v$ points so it has a resolution $\alpha_{i j}$. It is simple to verify that $\alpha_{i j}+\alpha\left(\beta_{i j}\right)$ resolves the original load $F$. This completes the induction for (ii), when $d=1$.

Dimension $d+1$. Assume that both (i) and (ii) have been verified up to dimension $d$, and consider a framework in a space of dimension $d+1$.

(i) Assume that $k$ joints $a_{1} \cdots a_{k}$ span a subspace of dimension $k-1 \leqslant d$, and that $F$ is a load in $(d+1)$-space applied to these joints. For convenience we assume that the joints lie in the subspace with $h$ th coordinate $\left(a_{i}\right)_{h}=0$. The equilibrium equations (for all coordinate directions $j$ ) are

$$
\sum_{i}\left(F_{i}\right)_{h}=0 \text { and } \sum_{i}\left(F_{i}\right)_{h}\left(a_{i}\right)_{j}-\left(F_{i}\right)_{j}\left(a_{i}\right)_{h}=0
$$

and these reduce to

$$
\sum_{i}\left(F_{i}\right)_{h}=0 \text { and } \sum_{i}\left(F_{i}\right)_{h}\left(a_{i}\right)_{j}=0 .
$$

However, since the $a_{i}$ are affinely independent, we conclude that all $\left(F_{i}\right)_{h}=0$. Thus all $F_{i}$ also lie inside the $d$-space. By our induction hypothesis, such a load must be resolved by the framework.

Now assume that $d+2$ joints $a_{0} a_{1} \cdots a_{d+1}$ span $(d+1)$-space, with an equilibrium load $F$ applied to these joints. By hypothesis each $F_{0 h}, 1 \leqslant h \leqslant d+1$, is resolved by the framework. Let the scalars $\beta_{i k}^{h}$ be the resolutions. Since the joints span $(d+1)$-space we also have scalars $\alpha_{h}$ which make $F_{0}=\sum_{h} \alpha_{h}\left(a_{0}-a_{n}\right)$. The new load $F-\sum \alpha_{h} F_{0 h}$ is now an equilibrium load on $d+1$ joints spanning $d$-space, and thus has a resolution $\alpha_{i j}$. A simple computation shows that $\alpha_{i j}+\sum_{h} \alpha_{h} \beta_{i j}^{h}$ is the required resolution of $F$. This completes case (i) in dimension $d+1$.

(ii) The joints of the framework span $(d+1)$-space. In (i) we proved (ii) when there are $d+2$ joints. Assume that we have confirmed that any load attached to $v$ joints is resolved, and $F$ is an equilibrium load applied to $v+1$ joints (clearly spanning the space). Let $a_{0} a_{1} \cdots a_{d+1}$ be an independent spanning set of joints. The entire argument given in case (i) now applies to give a resolution of the load $F$. This induction gives case (ii) in dimension $d+1$. 
If we read the proof carefully, we can deduce extra information about the size of the framework, and a process of adding new joints to frameworks. It is convenient to call a minimal statically rigid framework on an given set of joints an isostatic framework.

COROLlARY 2.2. If, to a statically rigid framework in $d$-space with $v>d$ joints, $a$ new joint is added with $d$ bars not all in an affine subspace, then the new framework is also statically rigid in $d$-space.

An isostatic framework in $d$-space with $v \geqslant d$ joints has $d \cdot v-d(d+1) / 2=E$ bars.

Proof. From our induction steps it is clear that the process for adding a joint $a_{0}$ provides the required resolutions for all $F_{0 h}$-and thus a resolution for any equilibrium load.

If we now re-examine the entire induction for $d$ independent joints in $d$-space, it becomes clear that we need the complete framework, with all pairs of joints joined. This gives $d(d-1) / 2$ bars. To add further joints by the process described above we need $d$ bars per joint. This gives final count

$$
E=d(v-d)+d(d-1) / 2=d v-d(d+1) / 2 .
$$

This number is clearly sufficient for static rigidity. Is it minimal? An isostatic framework provides bars $\{i, j\}$ such that the $F_{i j}$ form a basis of the space of equilibrium loads. This space is defined by $d+d(d-1) / 2=d(d+1) / 2$ linear equations on the space $\mathbf{R}^{d v}$. Thus it has dimension at least $d v-d(d+1) / 2$, and the structure defined above provides a spanning set. We conclude that the space of equilibrium loads has the stated dimension, and all isostatic frameworks on $v \geqslant d$ joints has the indicated size.

With a short inspection of this counting argument we can even give a converse to Corollary 2.2.

COROLlaRY 2.3. If a framework is statically rigid in $d$-space, with a joint having exactly d entering bars, then removing this joint and its bars leaves a smaller frameowrk which is statically rigid in dimension $d$.

Proof. We know that the bars of the first framework provide a spanning set of $F_{i j}$ for the equilibrium loads. Since the joint to be removed has $d$ bars, we know that $v \geqslant d+1$, and that removing the joint will drop a $d$-space of equilibrium loads-as well as $d$ bars from the spanning set. We conclude that the reduced framework still provides a spanning set of equilibrium loads.

If the bar forces $F_{i j}$ are linearly dependent then there is an assignment of scalars $\lambda_{i j}$ such that

$$
\sum \lambda_{i j} F_{i j}=0 \quad \text { (sum over bars). }
$$

This is equivalent to the equations for each joint $a_{i}$,

$$
\sum \lambda_{i j}\left(a_{i}-a_{j}\right)=0 \quad(\text { sum over } j \text { with }\{i, j\} \in B) .
$$


Such a nonzero assignment, a resolution of the zero load, is called a static stress. The equations say that there is a set of tensions $\left(\lambda_{i j}<0\right)$ and compressions $\left(\lambda_{i j}>0\right)$ in the bars which reach an equilibrium at each joint.

The set of all static stresses on a framework, together with the zero assignment, forms a vector space which we call the stress space. As a convenient shorthand we call the dimension of the space of stresses the number of stresses.

A framework with a stress can be statically rigid. The stress indicates a redundancy on the bars and a bar can be removed to break the dependence without changing the static rigidity. Such a framework is not isostatic (but it contains an isostatic subframework on the same joints).

We can make some of the generalities clearer by giving two simple results, on the line and the plane, which we will use regularly throughout the paper.

Proposition 2.4. A framework with all joints on the line is isostatic on the line if the bars form a connected tree of $v-1$ bars on $v$ points.

Proof. From Corollary 2.2, with $d=1$ we find that an isostatic framework has $v-1$ bars.

If the graph is not connected, then some component has $k$ bars on $\leqslant k$ joints. Since this piece is overcounted, it has a stress-and the entire framework is not isostatic, as noted above.

Similarly, if the framework is not a tree, then it includes a polygon which is overcounted, and contains a stress (Figure 1A).

We conclude that the framework is a tree.

To state the next result we need one further

Definition 2.4. A bar and joint framework is a triangulated polygon if it has $n \geqslant 3$ joints and these joints could be placed in the plane as a convex polygon $a_{1}, \ldots, a_{v}$ with the bars forming the cycle $\{1,2\},\{m-1, n\},\{n, 1\}$ plus a maximal set of noncrossing edges inside this polygon (Figures $1 \mathrm{~B}$ and $\mathrm{C}$ ).

Proposition 2.5. Any framework in the plane which is a triangulated polygon with no triangle of bars collinear is isostatic in the plane.
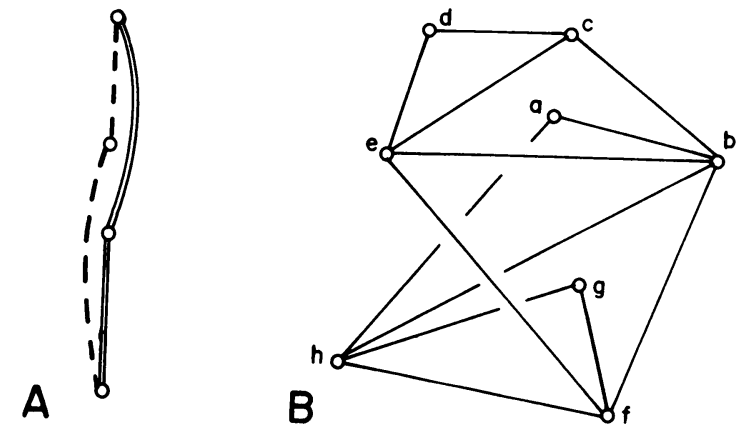

Figure 1 
Proof. Each triangulation with $v \geqslant 3$ has a 2 -valent joint. If we remove this joint we have a smaller triangulated polygon. When $v=3$ such a removal leaves a bar. We can now reverse this process. Begin with a bar, which is isostatic in the plane. By Corollary 2.2, repeated addition of 2-valent joints, with the two bars not collinear, preserves this isostatic property. Since no triangle is collinear, we have the desired insertions.

If we think of the bars of an isostatic framework as providing a basis for the space of equilibrium loads, then there are some simple substitution principles which we can observe. We will offer two variations-one for spanning sets and one for independent sets. The case of isostatic frameworks will then follow as a natural corollary.

THEOREM 2.6. If, in a statically rigid framework in $d$-space, the subframework of all bars on $k$ joints $a_{1} \cdots a_{k}$ is replaced by a new subframework on these joints which is statically rigid in the affine subspace spanned by the joints, then the modified framework is statically rigid in $d$-space.

Proof. A statically rigid framework gives a spanning set for the equilibrium loads. We are replacing some elements of this set by a new set which is guaranteed, by its static rigidity in the subspace, to resolve the loads $F_{i j}$ for bars being deleted. Thus we have a new spanning set.

THEOREM 2.7. If a framework in d-space is independent (has no stress) and a subframework among $k$ joints is statically rigid in the affine space spanned by the joints, and if a modified framework is created by replacing this subframework by a new independent subframework on these $k$ joints, then the modified framework is independent.

Proof. We can extend the original framework to an isostatic framework in whatever space the joints span. Similarly we can extend the new subframework to be inserted to a framework isostatic in the space of its joints. The new subframework will now preserve the static rigidity (Theorem 2.6) and will have the same count of bars as the isostatic subframework replaced. We conclude that after this replacement we have an isostatic framework containing the desired modified framework.

COROLlARY 2.8. If a framework is isostatic in $d$-space, with a subframework on $k$ joints statically rigid in the affine space of its joints, and this subframework is replaced by another subframework isostatic in the space of these joints, then the modified framework is isostatic in d-space.

This completes our static preliminaries. We now need an even briefer summary of the companion theory of infinitesimal kinematics.

Definition 2.5. An instantaneous motion of a bar and joint framework in $d$-space is an assignment of vectors in $d$-space, $\left\langle\ldots, v_{i}, \ldots\right\rangle$, to the joints such that, for each $\operatorname{bar}\{i, j\}$,

$$
\left(v_{i}-v_{j}\right) \circ\left(a_{i}-a_{j}\right)=0 \text {. }
$$


A bar and joint framework is infinitesimally rigid in $d$-space iff every instantaneous motion can be extended to an assignment of velocities to all points of space such that $(V(p)-V(q)) \circ(p-q)=0$ for each pair of points in space.

The motivation for these definitions lies in the idea of a motion which preserves the length of all bars: $(p-q) \circ(p-q)=c$. If this equation is differentiated we obtain the given equations on the velocities.

The given conditions on the velocities of an instantaneous motion are a set of linear equations in the $v_{i}$. If we construct the matrix for this system of homogeneous equations, the rigidity matrix, then the rows of the matrix are the vectors $F_{i j}$ we employed for the bars. Thus static rigidity was, in essence, a measure of the rank of the row space of the matrix, while infinitesimal rigidity measures the column rank (and nullity) of the matrix. It is not surprising that these two concepts of rigidity are equivalent. We state this result without proof. A general proof appears in [20, Theorem 4.3]. (To apply that result here, note that tensegrity frameworks include bar and joint frameworks as a special case (see §5).)

THEOREM 2.9. $A$ bar and joint framework is statically rigid in $d$-space iff it is infinitesimally rigid in $d$-space.

The failure of infinitesimal rigidity appears as an internal motion-a set of velocities which will not extend to a rigid or Euclidean motion of the entire space. When there are such internal motions, we would like to count "how many". The set of all infinitesimal motions of a framework forms a vector space, and the motions extending to rigid motions form a subspace. The difference in dimension between these two spaces is called the degree of internal freedom of the framework. Equivalently this is the dimension of a quotient space defined by calling two infinitesimal motions equivalent iff their difference is a rigid motion.

3. Convex polyhedra with triangulated natural faces. The original theorem of Cauchy, modified by Dehn, can be translated into statics to say that the natural edges of a convex polyhedron will not hold a static stress [8, 15]. An essential extension of this theorem, due to Alexandrov, translates to say that, even with addition of joints along the natural edges, and an arbitrary triangulation of the faces (including the added joints), the framework will not hold a static stress and thus is statically rigid as a bar and joint framework [1, 3].

We will begin, in this section, with a proof of a weaker form of Alexandrov's theorem - with no joints added along the natural edges, but the faces still triangulated. In the next section we will describe general techniques for adding vertices along the natural edges - techniques which apply to arbitrary statically rigid polyhedra.

We need definitions of these basic concepts.

DEFINITION 3.1. An abstract polyhedron is a finite set of vertices $v_{1}, \ldots, v_{n}$ and a set of faces $f_{1}, \ldots, f_{m}$ such that

(i) each face is a cycle of distinct vertices $f_{i}=\left\langle v_{i_{1}}, v_{i_{k}}\right\rangle, k \geqslant 3$, 
(ii) each edge (an adjacent pair of vertices $v_{i}, v_{j}$ in a face cycle) occurs in exactly two faces,

(iii) for each vertex $v_{j}$ there is a cycle of faces $\left\langle f_{j^{(1)}}, \ldots, f_{j^{(m)}}\right\rangle(m \geqslant 3$, no repetitions of faces) such that $v_{j}$ occurs on a common edge between adjacent faces of the cycle, and $v_{j}$ occurs on no other edges.

REMARK. This is the most general definition we could imagine [24]. Most of this paper will concentrate on spherical polyhedra (polyhedra where the graph of edges and vertices can be drawn on a sphere-or the plane with no edges crossing), but a number of results from $\$ \$ 4-7$ will apply more generally.

For this section we will concentrate on the convex polyhedra (Figure 2A).

Definition 3.2. A strictly convex polyhedron is a realization of an abstract polyhedron by distinct points $a_{1}, \ldots, a_{n}$ for the vertices such that:

(i) the joints affinely span 3-space,

(ii) for each face $f_{i}$ there is a single plane containing all vertices of the face (and affinely spanned by those vertices), which places all other vertices in a single open half-space.

It is a direct consequence of this definition that a strictly convex polyhedron is a spherical polyhedron (has a planar graph) and therefore satisfies Euler's formula $V-E+F=2$ (where $V, E$ and $F$ are the number of vertices, edges and faces of the polyhedron). Even if we now triangulate the convex polygons which form the faces, the graph will be spherical.

As a result we will be able to employ, in our later proof, a crucial lemma originally due to Cauchy. For an extensive discussion of the history and variations on this lemma we recommend [21].

LEMMA (CAUCHY) [15, p. 235]. Let L be a finite graph on the two-sphere with no loops and no region bounded by just two edges of $L$. Mark the edges of $L$ randomly with + and - and let $N_{a}$ be the number os sign changes as one circles around the vertex a. Let $N=\sum N_{a}$ be the total number of sign changes. Let $V$ be the number of vertices of $L$. Then $N \leqslant 4 V-8$.

In particular, it is impossible for every $N_{a}$ to be at least 4 .
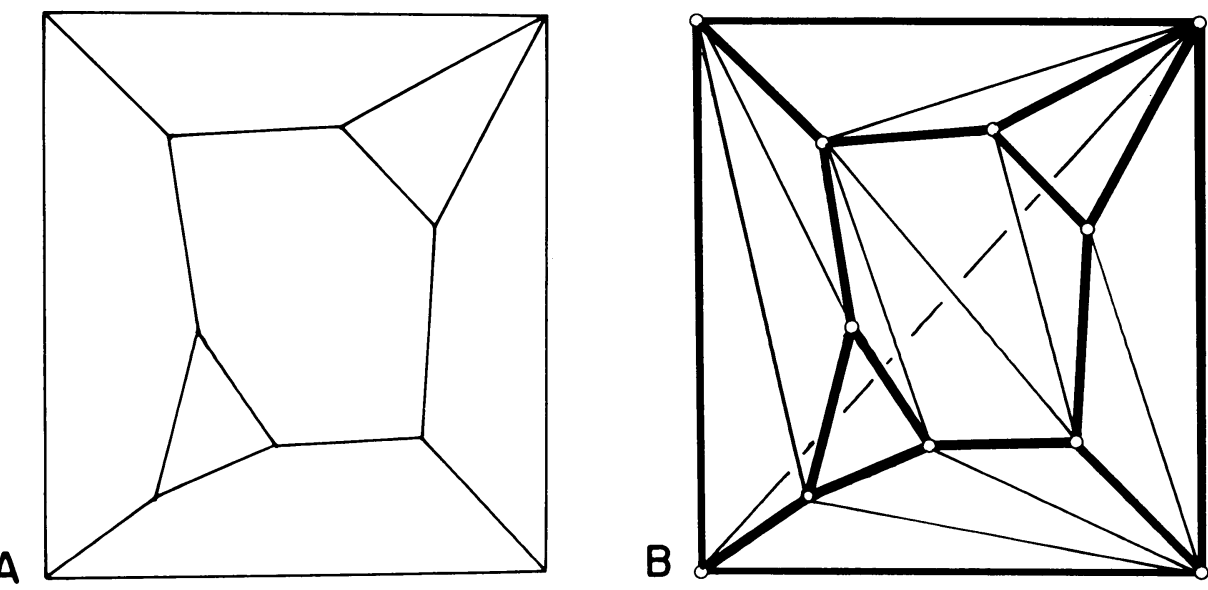

FIGURE 2 
We must now define our frameworks (Figure 2B).

DEFINITION 3.3. A strictly convex polyhedral framework is a bar and joint framework formed by

(i) placing a joint at each vertex of a strictly convex polyhedron,

(ii) placing a bar along each edge of the polyhedron,

(iii) triangulating each convex face of the polyhedron and placing a bar along each edge of this triangulation.

We note that for a triangulated sphere, with $V-E+F=2$ and $2 E=3 F$, we have $E=3 V-6$. Thus such frameworks are statically rigid (and infinitesimally rigid) iff there is no static stress.

Our proof will center on showing that there is no such stress. While previous workers have spoken in terms of infinitesimal motions, the pattern we use has a great deal in common with Cauchy's original proof. This similarity is based on a general isomorphism which translates infinitesimal motions, given as angular velocities between adjacent triangles, into scalars for a stress. This specific isomorphism was presented in $[\mathbf{1 1}, \S 4]$.

THEOREM 3.1. A strictly convex polyhedral framework is statically rigid in 3-space and is independent.

Proof. The proof will show that there is no static stress on the framework. We will proceed by induction on the number of joints $V$ in a subframework of any such strictly convex polyhedral frameworks.

If $V \leqslant 3$, then the subframework is some (or all) of a noncollinear triangle. We know that there is no stress on such a subframework.

Assume that all such subframeworks with $V \leqslant K$ joints do not have a static stress. We now take a subframework $H$ with $K+1$ joints. We further assume that there is a static stress $\left\langle, \lambda_{i j},\right\rangle$ on this subframework and proceed to derive a contradiction.

We first drop all bars with $\lambda_{i j}=0$. If this isolates any joint, we can drop this joint, and transfer the stress to a subframework with $V \leqslant K$ joints. This would be a contradiction, so we know that each joint $a_{0}$ still has a bar $\{0, j\}$ with $\lambda_{0 j} \neq 0$.

We now want to describe the number of sign changes of the $\lambda_{0 j}$ at the joint $a_{0}$. Clearly this must be an even number.

Case 1. If the number of sign changes is zero, then all $\lambda_{0_{j}}$ have the same sign ( $\lambda_{0 j}>0$ for simplicity). By the strict convexity there is a plane at $a_{0}$ which places all other vertices into a single open half-space. If $N$ is the normal to this plane we can assume $N \circ\left(a_{0}-a_{j}\right)>0$ for all bars $\{0, j\}$ entering $a_{0}$. Therefore

$$
N \circ\left(\sum_{j} \lambda_{0 j}\left(a_{0}-a_{j}\right)\right)=\sum_{j} \lambda_{0 j} N \circ\left(a_{0}-a_{j}\right)>0 .
$$

This contradicts the stress condition that $\sum_{j} \lambda_{0 j}\left(a_{0}-a_{j}\right)=0$, so we conclude that there must be at least two sign changes.

Case 2. Assume that there are two sign changes. For convenience we list all remaining edges entering $a_{0}$ in a cycle, $a_{0} a_{1}, a_{0} a_{2}, \ldots, a_{0} a_{m}$, with $\lambda_{0 i}>0$ for $1 \leqslant i \leqslant k$ and $\lambda_{0 j}>0$ for $k<j \leqslant m$. 
Consider the (unique) plane defined by $a_{0} a_{1} a_{k}$, with normal $N$. By the convexity of the original polyhedron we have

$$
N \circ\left(a_{0}-a_{i}\right) \geqslant 0, \quad 1 \leqslant i \leqslant k, \quad \text { and } \quad N \circ\left(a_{0}-a_{j}\right) \leqslant 0, \quad k<j \leqslant m,
$$

and thus

$$
\lambda_{0 i} N \circ\left(a_{0}-a_{i}\right) \geqslant 0 \text { for all } 1 \leqslant i \leqslant m
$$

We conclude that

$$
N \circ\left(\sum_{i} \lambda_{0 i}\left(a_{0}-a_{i}\right)\right)=\sum_{i} \lambda_{0 i} N \circ\left(a_{0}-a_{i}\right) \geqslant 0 .
$$

Since we started with a stress we conclude that $N \circ\left(a_{0}-a_{i}\right)=0$ for all $1 \leqslant i \leqslant m$ - and thus all the remaining bars entering $a_{0}$ are coplanar. Moreover, to obtain an equilibrium at $a_{0}$ with no pair of collinear bars, there must be at least three coplanar bars remaining. This is only possible if $N$ defines a face plane and all remaining bars lie in this face plane.

We will now show that this is also impossible by showing how to remove the joint $a_{0}$, with all entering bars in a single face, without removing the stress.

Consider the remaining subframework $P$ in this face plane $-P$ contains all the bars in that plane with $\lambda_{i j} \neq 0$ (Figure $3 \mathrm{~A}$ ). It is a subframework of a triangulated polygon, so it does not resolve the entire original stress. It must be attached to other parts of $H$ at joints $b_{1}, \ldots, b_{r}$.

We will define a plane equilibrium load at these joints. Consider the plane equilibrium load

$$
G=\sum \lambda_{i j} F_{i j} \quad(\text { sum over }\{i, j\} \text { in } P) .
$$

At all vertices except $b_{1}, \ldots, b_{k}$ we have all entering bars of the stress, so $G$ gives a zero load at these vertices. At each $b_{i}$ we have defined $G(i)$, the sum of the forces in bars of $P$, and this must exactly match $H(i)$, the sum of the forces in bars at $b_{i}$ not in $P: H(i)+G(i)=0$, with at least some $G(i) \neq 0$ (Figure 3B).

We now replace all of $P$ by a new subframework $P^{\prime}$ on the joints $b_{1}, \ldots, b_{r}$. If $r=2$, we use the bar $b_{1} b_{2}(r \neq 1$ since no single, nonzero force $H(i)$ can be in

A

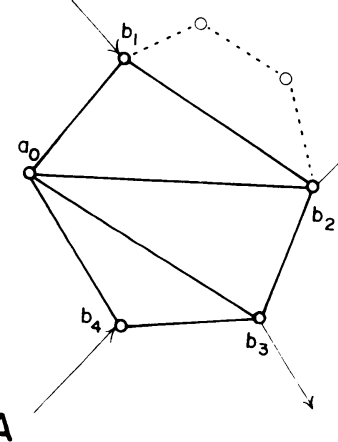

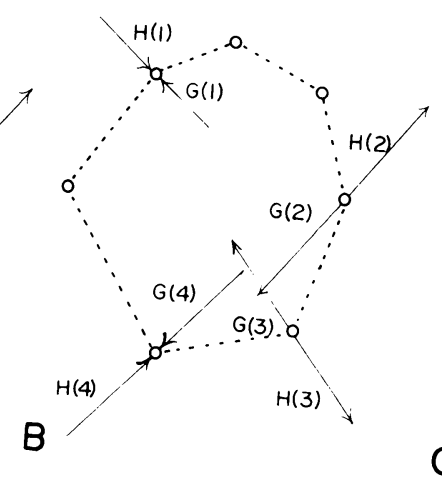

FigURe 3

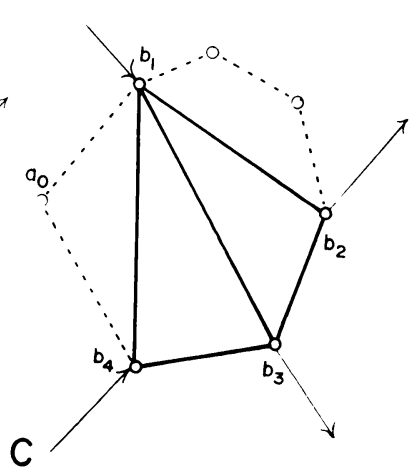

C 
equilibrium). If $r \geqslant 3$ we have some vertices of a convex polygon, and we take any triangulation of this smaller polygon. In both cases, $P^{\prime}$ is part of some triangulation of the original face. Since $P^{\prime}$ is plane-statically rigid, it will resolve the equilibrium load $G$ with scalars $\beta_{i j}\left(\{i, j\}\right.$ a bar in $\left.P^{\prime}\right)$.

If we now take $H$ as $H-P+P^{\prime}$, these scalars $\beta_{i j}$ on $P^{\prime}$ and $\lambda_{i j}$ on the other bars will form a nontrivial stress: At $b_{i}$ we have

$$
\sum_{h} \lambda_{i_{h}}\left(b_{i}-a_{h}\right)+\sum_{j} \beta_{i_{j}}\left(b_{i}-b_{j}\right)=H(i)+G(i)=0 .
$$

This substitution has transferred the stress to a smaller subframework omitting $a_{0}$ (Figure $3 \mathrm{C}$ ) - a situation which contradicts our induction assumption. Thus no joint $a_{0}$ can have exactly two sign changes.

Case 3. Every joint of $H$ has at least 4 sign changes in the stress. If we now use the $\lambda_{i j}$ to assign + and - signs to edges of $H$ we have an assignment contracting Lemma $\mathrm{T}$.

We conclude that no stress exists on $H$, and the induction is complete.

4. Inserting joints on the natural edges. Alexandrov gave an extension of Theorem 3.1 in which vertices are inserted along the interior of the natural edges of the convex polyhedron, and the faces are triangulated. This result was obtained by an extended analysis of "sign changes at a vertex" [1]. An English translation of this proof appears in $[3, \S 6]$. Connelly has recently observed that this extension can also be obtained by a detailed analysis of the infinitesimal motions of a triangulated disc [10, Remark 6.1].

We will use the static insertions and replacements from $\$ 2$ to show how to add vertices on the interior of natural edges of any statically rigid polyhedra. These techniques make no use of convexity of the polyhedra, convexity of the faces, or any other details about the polyhedral framework.

In $\$ 7$ we will give some examples of nonconvex statically rigid polyhedral frameworks. In $\$ 9$ we describe, briefly, the current situation on identifying other classes of statically rigid polyhedral frameworks. It seems helpful to present these insertions in enough generality to apply to both the current and the anticipated classes of frameworks.

DEFINITION 4.1. A strict polyhedron in 3-space is a realization of an abstract polyhedron by distinct points $a_{1}, \ldots, a_{n}$ for the vertices such that:

(i) the joints $a_{1}, \ldots, a_{n}$ affinely span 3 -space,

(ii) for each $F_{i}$ there is a single plane containing all vertices of the face (and affinely spanned by these vertices),

(iii) for each edge, the two faces at this edge have different face planes.

Definition 4.2. A strict polyhedral framework is a bar and joint framework built by

(i) placing a joint at each vertex of a strict polyhedron,

(ii) placing a bar along each natural edge of the polyhedron,

(iii) placing any desired additional bars between pairs of joints at two vertices of the same face. 
REMARK. Strictly convex polyhedral frameworks are clearly examples of strict polyhedral frameworks. However, we have eliminated all constraints on the form of the subframework in each face, as well as the general form of the polyhedron. In Figure 4A we show a nonconvex spherical polyhedron (each polygon is a plane face). Figures $4 \mathrm{~B}$ and $\mathrm{C}$ show possible strict polyhedron frameworks built from this polyhedron.

THEOREM 4.1. If a statically rigid strict polyhedral framework is modified by

(i) adding new joints along the natural edges of the strict polyhedron,

(ii) replacing the original subframework on each face by a new plane statically rigid subframework on all joints of this face (including any new joints on the edges of this face),

then this modified bar and joint framework is statically rigid in space.

Proof. We first add the new joints while keeping the framework statically rigid at each stage.

For each new joint $b$ we have two adjacent face planes $F_{1}$ and $F_{2}$ meeting at the natural edge. We add the joint $b$ as a 3-valent joint connected to one end of the natural edge, and to two joints off this line-one in each of the faces $F_{1}$ and $F_{2}$. Since the face planes are different, we have added a 3-valent joint which is not flat and the new framework is statically rigid, by Corollary 2.2 .

Assume that all of the new joints have been added by this process. We now take the subframework in a face (including any new joints on the edges of this face) and replace it by a new plane-statically rigid framework on these joints. By Theorem 2.6 the result is a new statically rigid framework.

When this process is completed for all faces, the desired framework has been produced and it is statically rigid.

It is clear from the proof that there is a natural converse, provided that the original framework has plane-statically rigid faces.

THEOREM 4.2. If a strict polyhedral framework is modified by

(i) the addition of joints along the natural edges,

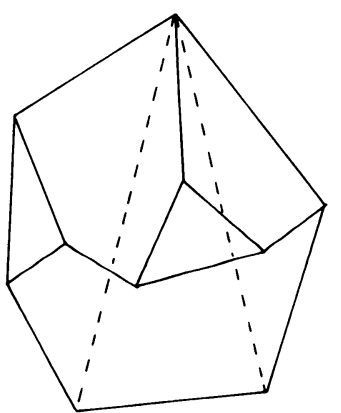

A

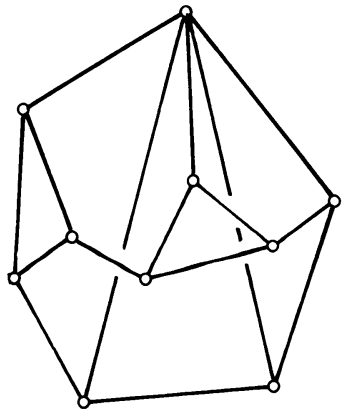

B

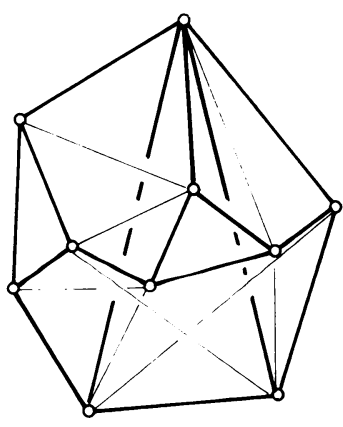

C

FIGURE 4 
(ii) the replacement of the subframework on each face by a new plane subframework, and this modified framework is statically rigid in space, and if the original framework had a subframework on each face which was plane-statically rigid, then the original framework was also statically rigid in space.

PROof. We will simply reverse the steps of the previous proof.

For each added joint on a natural edge we preassign three edges-one along the natural edge to a natural vertex and one across each adjacent face.

We now replace the final subframework in a face $P_{i}$ with the original subframework $F_{i}$ of the strict polyhedral framework, plus two bars for each added joint, these along the two preassigned edges which lie in the face plane. Since the original subframework $F_{i}$ was plane statically rigid, and the addition of two valent joints (with the bars not collinear) preserves plane-static rigidity, this entire replacement preseves the spatial rigidity of the framework.

When this process is completed for all the faces, we have each added joint as a three-valent joint (the three bars not coplanar). By Corollary 2.3 these can be deleted to leave a statically rigid framework - the original strict polyhedral framework!

If we restrict our attention to triangulated spherical polyhedra, this result takes a simple form. We recall that for such a polyhedron $E=3 V-6$, so static rigidity is equivalent to independence of the bars. Secondly, any triangulation of a polygon with no collinear triangles is independent and plane-statically rigid. We assume that any triangulation has no triangle collinear.

COROLLARY 4.3. Given a strict polyhedral framework which is built on a spherical polyhedron with each face triangulated by bars, and a modified framework formed by

(i) the addition of joints along the natural edges,

(ii) the replacement of the subframework on each face by a triangulation of the face polygon with the added joints,

then the modified framework is independent (and statically rigid) iff the original strict polyhedral framework was independent (and statically rigid).

Proof. The proof proceeds from Theorems 4.1 and 4.2. We need only add the observations that all facial frameworks are isostatic in the plane, and that, for the triangulations of a sphere independent is equivalent to static rigidity.

We note that the "strong form" of Alexandrov's theorem is a particular case of Corollary 4.3, where the original strict polyhedral framework was convex, and therefore statically rigid.

COROLlARY 4.4. If a strictly convex polyhedra is built as a bar and joint framework by

(i) the placement of joints at the natural vertices and along the natural edges,

(ii) the placement of bars on the natural faces to form the polygon on the joints around the natural edges,

(iii) the placement of bars on the natural faces to follow a triangulation of the new face polygon, then the bar and joint framework is statically rigid (and independent) in space. 
We want to generalize Corollary 4.3 to eliminate the condition that all face frameworks are triangulations. However, we cannot simply use arbitrary planeisostatic frameworks in the faces and expect to obtain an isostatic framework. Figure 5 illustrates the problem (which does not depend on any joints interior to edges). In Figure 5A we show a nice isostatic (convex) strict polyhedral framework. In Figure $5 \mathrm{~B}$, each new face is still plane-isostatic, but the overall framework is statically rigid and overbraced ( $E=3 V-5)$. There is a stress, which we illustrate in Figure 5C (using dashed lines for tension, $\lambda<0$, and double lines for compression, $\lambda>0$ ). If we remove one bar (Figure 5D), the new framework is isostatic in space, but one face has an underbraced subframework.

The key problem is the missing natural edge. Corollary 4.3 has nice generalizations provided the natural edges are "present"- the subframework on the natural edge is isostatic in the line (i.e. forms a tree).

COROLlaRY 4.5. Given a statically rigid strict polyhedral framework built on a spherical polyhedron and a modified framework formed by the addition of joints along the natural edges, and the replacement of the subframework in each face by a subframework on all the joints of the face, such that each natural edge has a subframework isostatic on the line, then

(i) the modified framework is independent iff the subframework in each face of the modified framework is independent,

(ii) the modified framework is statically rigid iff the subframework in each face of the modified framework is plane-statically rigid.

Proof. (i) Clearly if the modified framework is independent, then the subframework on each face must be independent.

Conversely, assume that the subframework on each face is independent. We can add bars to each face to make this subframework plane-statically rigid and independent. When this is finished each face $F_{i}$ has $E_{(i)}=2 V_{(i)}-3$ bars and on each natural edge $(i, j)$ we have $E_{(i, j)}=V_{(i, j)}-1$ bars. Since these counts exactly match the counts for a triangulated sphere, we have $E=3 V-6$. Since each face is statically rigid this extension of the modified framework is statically rigid. We conclude that this extension is independent and therefore the modified framework was independent.

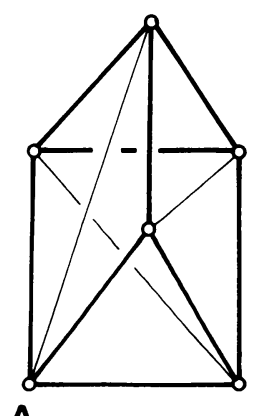

A

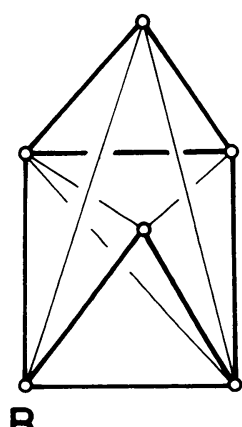

B

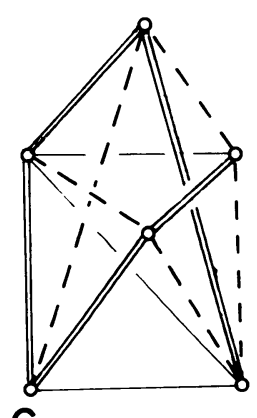

$\mathrm{C}$

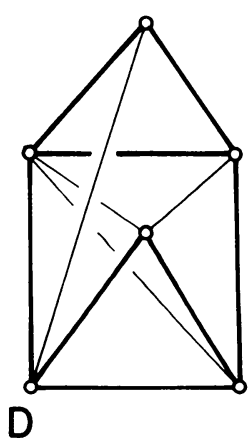

FIGURE 5 
(ii) Clearly, if each face is plane-statically rigid then, by Theorem 4.1 , the entire modified framework is statically rigid.

Conversely, assume that the entire modified framework is statically rigid, and that the subframework on each natural edge is a tree on the joints. For the subframework on each face the bars along the natural edges form an independent set, and we can delete just enough bars from the interior of the face to make the subframework independent. Since each deletion removes a stress from the face (and thus from the entire structure) this leaves a statically rigid framework $E \geqslant 3 V-6$. For each face $F_{i}$ we have $E_{(i)} \leqslant 2 V_{(i)}-3$ (with a nonnnegative deficit $K(i)$ ) and for each natural edge we have $E_{(i, j)}=V_{(i, j)}-1$. By a simple comparison with a triangulation of the same sphere (using the facial polygons) we have

$$
E+\sum K(i)=3 V-6 .
$$

Since we also have $E \geqslant 3 V-6$ we conclude that each $K(i)=0$ and the faces are all still statically rigid.

The assumption that each natural edge forms a tree is essential as the following result illustrates.

COROLlARY 4.6. Given a statically rigid strict polyhedral framework on a spherical polyhedron, and an independent modified framework formed by the addition of joints along the natural edges and the replacement of the subframework in each face by an independent, plane-statically rigid framework on all the joints of the face, then the subframework on each natural edge is a tree.

Proof. The proof is a simple counting argument, based on a comparison with a triangulation of the sphere. We will omit it.

5. Tensegrity frameworks. To this point all of our frameworks have used bars to join pairs of joints. However, there has been an interest in frameworks using cables - members which prevent any increase in the distance between the joints, but place no restriction on a shortening of the distance $[10,14]$.

It is a simple matter to reconstruct our theory of static rigidity to incorporate the cables, members which only support tension $\left(\lambda_{i j} \leqslant 0\right)$, and also to incorporate struts, members which only support compression $\left(\lambda_{i j} \geqslant 0\right)$, as well as the bars which permit a general $\lambda_{i j}$. This general static theory for tensegrity frameworks (as well as the equivalent theory of infinitesimal motions) has been presented in [20].

We will simply summarize the essential definitions and properties here, and then give the applications to statically rigid polyhedral frameworks.

Definition. 5.1. A tensegrity framework in $d$-space is a set of joints $\left(a_{1}, \ldots, a_{v}\right)$ (points in $d$-space) and three pairwise, disjoint collections of unordered pairs $\{i, j\}$ of indices for joints $B$ (the bars), $C$ (the cables) and $S$ (the struts) such that for $\{i, j\} \in E=B \cup C \cup S, a_{i} \neq a_{j}$.

An equilibrium load on a tensegrity framework in $d$-space is an assignment of forces to the joints $F=\left(F_{1}, \ldots, F_{v}\right), F_{i} \in \mathbf{R}^{d}$, such that the forces are in equilibrium in space.

A resolution of an equilibrium load by a tensegrity framework is an assignment of scalars $\lambda_{i j}$ for each $\{i, j\} \in E$ such that $\lambda_{i j} \leqslant 0$ for $\{i, j\} \in C$, and such that 
$\lambda_{i j} \geqslant 0$ for $\{i, j\} \in S$, and for each joint $a_{i}$

$$
\sum \lambda_{i j}\left(a_{i}-a_{j}\right)+F_{i}=0 \quad(\text { sum over } j \text { with }\{i, j\} \in E) .
$$

A tensegrity framework is statically rigid in $d$-space iff the framework has a resolution for each equilibrium load in $d$-space on the framework.

It is clear that a bar and joint framework is a particular case of a tensegrity framework, with $C=S=\varnothing$. In this case all definitions coincide with our previous definitions.

Without pursuing detailed definitions, we note that infinitesimal rigidity and static rigidity are equivalent for tensegrity frameworks [20, Theorem 4.3].

As a practical measure, we normally determine the static rigidity of a tensegrity framework $T$ by a careful comparison with the bar and joint framework $\bar{T}$ which has the same joints, but replaces all cables and struts by additional bars.

THEOREM 5.1 [20, THEOREM 5.2]. A tensegrity framework $T$ is statically rigid in $d$-space iff the bar and joint framework $\bar{T}$ on the same graph is statically rigid in $d$-space and the bar and joint framework has a proper stress: a stress $\left(\ldots, \lambda_{i j}, \ldots\right)$ with $\lambda_{i j}>0$ for each $\{i, j\} \in S$ and $\lambda_{i j}<0$ for each $\{i, j\} \in C$ and $\lambda_{i j}$ arbitrary for $\{i, j\} \in B$.

REMARK. It is a direct consequence of this characterization that we can switch cables and struts without any change in the static rigidity of a framework (just multiply the proper stress by -1 ).

If a spatial framework is statically rigid with even one cable or strut, then it must have a stress and contain edges which form a basis for the equilibrium loads. As a result it must have at least $3 V-5$ members. Thus a statically rigid tensegrity framework on the surface of a spherical polyhedron, with at least one cable or strut, must have crossing members!

Since statically rigid tensegrity frameworks resolve the same loads as statically rigid bar and joint frameworks all of the substitution principles on static rigidity from $\$ 2$ apply directly to tensegrity frameworks. As a result, we can simply translate Theorem 4.1 to this setting.

THEOREM 5.2. If a statically rigid strict polyhedral framework (bar and joint) is modified by

(i) the addition of new joints along the natural edges of the strict polyhedron,

(ii) the replacement of the subframework on each face by a new plane-rigid tensegrity subframework on all joints on this face, then the modified tensegrity framework is statically rigid in space.

What are some possible patterns for plane-statically rigid tensegrity frameworks on a face? In Figure 6 we illustrate several ways to make a convex polygon statically rigid by adding interior cables [20, Theorems 6.3, 6.4]. (Struts are drawn as double lines and cables are drawn as dashed lines.) Because each face can be rigidified in this way, we have the following corollary which answers a conjecture of Grünbaum [14, Conjecture 4]. 
COROLlaRY 5.3. If a strict polyhedral framework is statically rigid with all faces convex, then it is possible to place bars on the natural edges and place sufficient cables on each face ( $K-2$ cables for each $K$-gon $K>3$ ), so that the new tensegrity framework is statically rigid in space.

For spherical polyhedra, this is the best that we can do. It is a simple observation that a nonconvex polygon cannot even support a stress with only cables across its interior (and thus by Theorem 5.1 it will not be plane-statically rigid). Since a sphere requires that each face be statically rigid we are finished.

Of course there are patterns, with some bars (or struts) "across" the faces which will make nonconvex faces plane-statically rigid (Figure 7A). It is even possible to find configurations which include joints along the natural edges (Figure 7B), so Corollary 5.3 was not vacuous.

For other types of polyhedra (such as the torus), it is possible to build tensegrity frameworks without any crossing members (i.e., within triangulations of the surface) since a triangulation of the torus will have $E=3 V>3 V-6$. The form of these stresses can only be determined by a direct analysis of the original triangulated surface.

6. Inserting facial joints. In $\S 4$ we gave a careful analysis of the static rigidity and the independence of polyhedral bar and joint frameworks with new joints added along the natural edges. What happens if we add new joints inside a face?

If we add such a joint to a statically rigid framework as a 3-valent joint then we know, by Corollary 2.2, that the new framework is statically rigid iff the three new bars are not coplanar. Thus, when we add a facial joint we must insert at least one bar off the face (and therefore off the surface of the polyhedron), if we want to obtain static rigidity (Figure 8A). Put in other terms, if we insist that all bars lie in

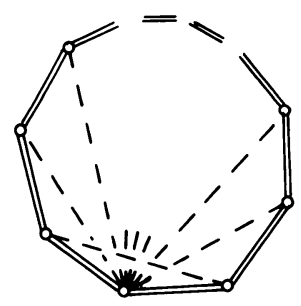

A

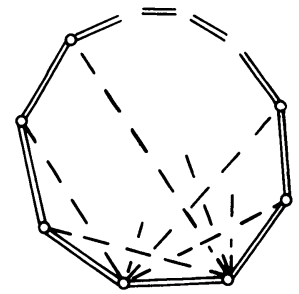

B

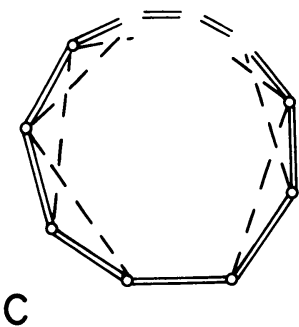

Figure 6

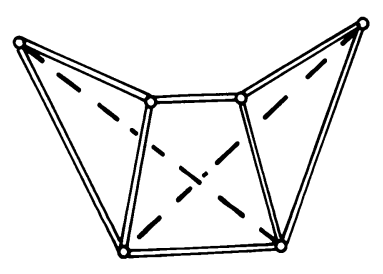

A

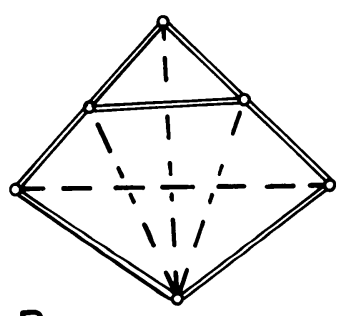

B

FIGURE 7 
the faces, then any facial joint will have an infinitesimal "vibration" normal to the face which fixes all other joints (Figure 8B).

THEOREM 6.1. If a statically rigid strict polyhedral framework is modified by

(i) the addition of joints along the natural edges,

(ii) the addition of $k$ joints, each identified with a face, and lying in the plane of this face,

(iii) the replacement of the subframework on each face by a new plane-statically rigid subframework on all joints of the faces (including new facial joints identified with the face and new joints on its natural edges),

then the modified framework has $k$ degrees of internal freedom, and these internal motions are generated by fixing all joints on natural edges, and moving each of the $k$ facial vertices normal to its face.

Proof. We first add the joints on the natural edges, as in Theorem 4.1. We then add the facial joints using two bars (not collinear) in the face and one bar off the face, connecting the new joint to a natural vertex. If we now do the replacements of the facial subframeworks, retaining these extra bars to the facial joints which are off the face plane, then the new framework is statically rigid by Theorem 2.6.

If we now delete the $k$ bars which run from facial joints but off the faces, then we introduce at most $k$ degrees of internal freedom (removing $k$ rows can lower the rank of a matrix by at most $k$ ). The $k$ vibrations described for these vertices are clearly independent internal motions (and no linear combination is a rigid motion) so we conclude that we have exactly $k$ degrees of internal freedom.

REMARK 1. We now know that such frameworks with facial joints and all bars in the faces are never infinitesimally rigid. Are they finitely rigid? If the final framework is independent, and there is an infinitesimal motion, then the framework is a mechanism. In fact, any framework which is independent and is not infinitesimally rigid is a mechanism [2, Theorem 4.1]. For example, consider a square based pyramid (Figure 9A). We add a vertex to the base and keep the plane framework independent (Figure 9B). The entire framework will be independent, and it will have a finite motion which begins with a motion of the added vertex normal to its face.

REMARK 2. Connelly has proven that if we add facial joints and an arbitrary (noncrossing) triangulation of the faces on a convex polyhedron, then the resulting

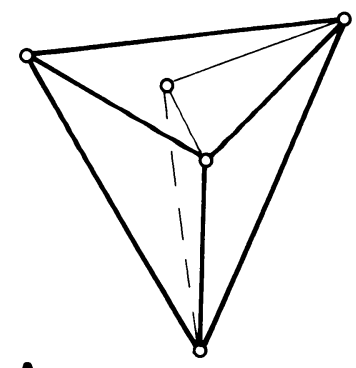

A

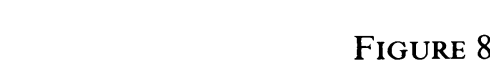

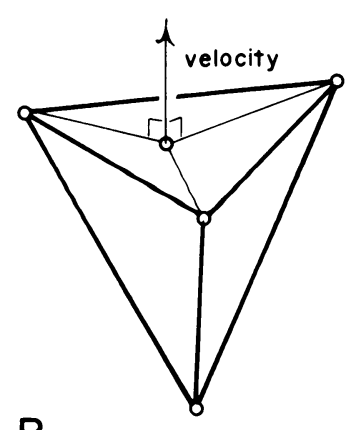

B 
bar and joint framework is second-order rigid and hence has no finite motions [10, Theorem 6]. Without going into the details of this concept, or the proof, we simply note that the essential tools Connelly uses are:

(i) the infinitesimal rigidity of the underlying polyhedral framework with no facial vertices, and a facial triangulation,

(ii) the behavior of triangulated convex discs as interior joints vibrate out of the plane,

(iii) the fact that the only internal motions of the facial joints are vibrations perpendicular to their plane.

Using Theorem 6.1 we can start with any statically rigid strict polyhedral framework and extend his results to obtain the second order rigidity of arbitrary retriangulations (with facial vertices) of any convex faces and retriangulations without facial vertices on other faces.

What happens to the stresses as we insert these additional facial vertices? We offer the following general theorem, which extends Corollaries 4.3 and 4.5.

THEOREM 6.2. Given a statically rigid strict polyhedral framework built on a spherical polyhedron, and a modified framework formed by

(i) the addition of joints along the natural edges and in the interior of the faces,

(ii) the replacement of the subframework in each face by a new bar and joint framework, such that the final subframework on each natural edge is line-statically rigid and independent,

then the space of stresses on the entire framework is the direct sum of the spaces of stresses on each of the faces.

Proof. We observe that each stress $\left\langle w_{k}\right\rangle$ which is nonzero only on the subframework of a face $F_{k}$ is a stress on the entire framework (with zero coefficients on all other bars). Are these stresses independent? Consider a set of facial stresses (one from each of a set of faces) which are presumed dependent: $\Sigma\left\langle w_{k}\right\rangle=0$. Each bar $\{i, j\}$ interior to a face occurs in only one $w_{k}$ so this $w_{k}$ is zero on the bar. We then have each $w_{k}$ supported by the bars on the natural edges of the face. Since each natural edge has been replaced by a tree, this subframework is independent and each $w_{k}=0$. We have a correct direct sum of stresses on the faces.

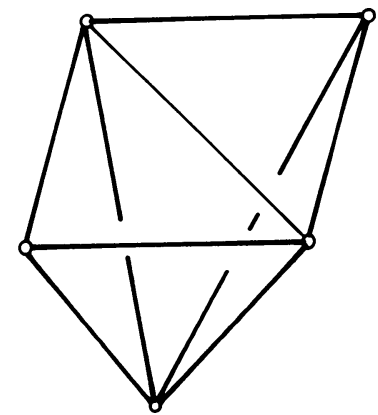

A

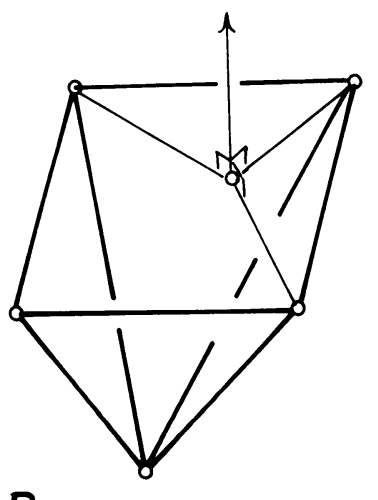

B

Figure 9 
Now, must every stress on the entire modified framework be in this direct sum?

We first delete from the interior of each face $F_{k}$ a sequence of bars, each one of which removes a facial stress (and thus a stress on the entire framework). Since the tree structure on the natural edges is independent, we can exactly eliminate the facial stresses this way.

The question remains - is there any stress on this reduced framework $H$ ?

To show that $H$ is independent we will extend it to a statically rigid, independent framework. We add bars to the interior of the facial subframeworks to make these plane-statically rigid (and independent) and add one more bar from each facial joint to a natural joint off its plane. This is a statically rigid framework $H^{\prime}$. We now replace each face by a plane-statically rigid, independent framework, using the same trees on the natural edges and only 2 bars to each facial vertex. This keeps the same count of bars on this (and every other) face. When this process is completed for all faces we delete the 3-valent facial vertices, obtaining, by Corollary 4.3, an independent framework with $E=3 V-6$. Thus $H^{\prime}$ is independent, as was $H$.

This proves that all stresses were removed when we removed the facial stresses.

If the final framework in Theorem 6.2 is a triangulated sphere, then we can give an exact count of the stresses.

COROLlARY 6.3. If a statically rigid strict polyhedral framework is built on a spherical polyhedron, and this framework is modified by

(i) the addition of joints on the natural edges and $K$ joints on the interior of faces,

(ii) the replacement of the subframework on each face by a triangulation of the face polygon (with all added joints and no collinear triangles), then the modified framework has a K-dimensional space of stresses, and each face $F_{h}$ with $K(h)$ facial joints has a $K(h)$-dimensional space of stresses in its subframework.

Proof. For a triangulated sphere we have $E=3 V-6$. Since each subframework on a face is plane-statically rigid, we know that we have $k$ degrees of internal freedom-and hence a $k$-dim space of stresses.

For each face $F_{h}$ with $K(h)$, internal joints, it is easy to verify that a triangulation has $2 V-3+K(h)$ edges and thus $K(h)$ stresses on this face. This gives all the $K=\sum K(h)$ stresses, as required.

REMARK. If we triangulate a nonspherical polyhedron, then we will always obtain stresses-since $E>3 V-6$. Can the special stresses, due to facial joints, be singled out when the original strict polyhedral framework was statically rigid? Yes. This characterization will be presented in our paper [27], after we have discussed the appropriate "path condition" needed for this characterization (and for motions of a panel structure).

From Theorem 6.2 we can conclude the independence of a modified framework, on a spherical polyhedron, with the facial substructures independent, the subframework on each natural edge line-statically rigid, and the original strict polyhedral framework statically rigid. There is a stronger result, which we will employ in the work on panel structures. (As noted in [11, Proposition 4.1], a panei structure on a 
polyhedron is infinitesimally rigid if the skeleton of its hinges is independent as a bar and joint framework.) Since the proof of this result on independence follows the same pattern as other theorems here, we present the result now.

THEOREM 6.4. If an independent strict polyhedral framework is modified by

(i) the addition of joints along the natural edges with a bar across each adjacent face and the joint splitting the natural edge,

(ii) the addition of joints inside any face of the strict polyhedron, each with two noncollinear bars in the face, to previously present joints,

(iii) the replacement of the subframework on any plane-statically rigid faces (or a plane-statically rigid section of the face) by an independent subframework including $a$ line-statically rigid subframework on each natural edge, then the modified framework is independent.

Proof. We begin by adding bars (anywhere) to make the original framework statically rigid and independent.

We now add the new joints, in the fashion of Theorem 6.1 creating a statically rigid, independent framework. The final replacement of faces which began as plane-statically rigid, will preserve static rigidity and preserve the count of bars. Therefore we have created a statically rigid, independent framework containing the modified framework.

REMARK. The most interesting examples of frameworks which we hope to find independent are subframeworks of a triangulation of the polyhedron. In this case the subframework in each face has a planar graph, and we have a geometric characterization of the independent facial subframeworks: A plane framework with a planar graph is independent iff the edges do not contain the central projection of a strict polyhedron from 3-space [23, Corollary 3.5]. This same result, originally observed by Maxwell [16], can be used to explain the patterns of stress used to produce the plane-statically rigid tensegrity frameworks described in $\$ 5[23, \S 4]$.

7. Projective transformations. It is an old result, first observed more than a century ago, that the properties of static rigidity and independence are not changed by a projective transformation of space. This has been presented elsewhere both in Euclidean language [20, Theorem 5.10] and in a directly projective setting [11, §2]. We will summarize the situation informally. Our object is to describe how additional statically rigid frameworks can be created by projecting those studied in \$§3-6. These will provide concrete examples on nonconvex statically rigid strict polyhedral frameworks.

To understand projective transformations we must first extend Euclidean 3-space to projective 3-space by adding a plane at infinity. Algebraically, we use homogeneous coordinates: each finite point $\bar{p}=\left(p_{1}, p_{2}, p_{3}\right)$ is written as $p=$ $\left(\lambda p_{1}, \lambda p_{2}, \lambda p_{3}, \lambda\right)$ (for any $\left.\lambda \neq 0\right)$, and the points at infinity by $q=\left(q_{1}, q_{2}, q_{3}, 0\right)$ (at least one of $q_{1}, q_{2}, q_{3} \neq 0$ ). In this algebra, two sets of coordinates $q$ and $q^{\prime}$ represent the same point (finite or infinite) iff $q^{\prime}=\lambda q$ for some $\lambda \neq 0$. (No point has coordinates $(0,0,0,0)$.) 
In these homogeneous coordinates three points $p, q$ and $r$ are collinear iff their coordinates are linearly dependent. This condition defines lines for points at infinity as well.

A nonsingular projective transformation, or a collineation, is a transformation which takes points to points, so that lines map to lines, and 4 noncopolanar points map to 4 noncoplanar points. In algebraic terms each collineation comes from a nonsingular linear transformation on the coordinates (from $\mathbf{R}^{4}$ to $\mathbf{R}^{4}$ ).

If we apply a projective transformation to the joints of a framework, and all the images of the joints are new finite points, then it is a simple matter to reconstruct the projected framework by taking Euclidean names for the images of the joints and taking the same bars $\{i, j\}$. These bars will, of course, be visualized as the line segments which are now finite.

It is less obvious, but true, that this transformation can be extended to carry static stresses to static stresses, and thus to preserve static rigidity. The following theorem summarizes this projective invariance [20, Theorem 5.10].

THEOREM 7.1. A bar and joint framework is statically rigid (respectively independent, stressed ) iff a projective transformation of the framework is statically rigid (respectively independent, stressed).

For tensegrity frameworks there is one additional fact. When we project a cable $\{a, b\}$ so that the new plane at infinity cuts the interior of the old cable, then this "infinite cable" should be replaced by a finite strut. Similarly, an "infinite strut" is replaced by a finite cable. This reversal reflects the pattern of tensions and compressions when a stress is projectively transformed. This pattern is illustrated, for plane frameworks, in Figure 10 and is reflected in the following result [20, Theorem 5.10].

COROLlaRy 7.2. Consider a tensegrity framework $T=\{V ; B, C, S\}$ and projective map $P$ taking the joints of $T$ to the joints of $T^{\prime}=\left\{V^{\prime} ; B, C^{\prime}, S^{\prime}\right\}$ (with indices preserved) such that a cable $\{i, j\} \in C$ (resp. strut $\{i, j\} \in S$ ) for which the line segment $\left[a_{i} a_{j}\right]$ intersects the plane $H$ mapped to infinity by $P$ is replaced by a strut $\{i, j\} \in S^{\prime}$ (resp. cable), and the remaining members are unchanged. Then $T$ is statically rigid iff $T^{\prime}$ is statically rigid.

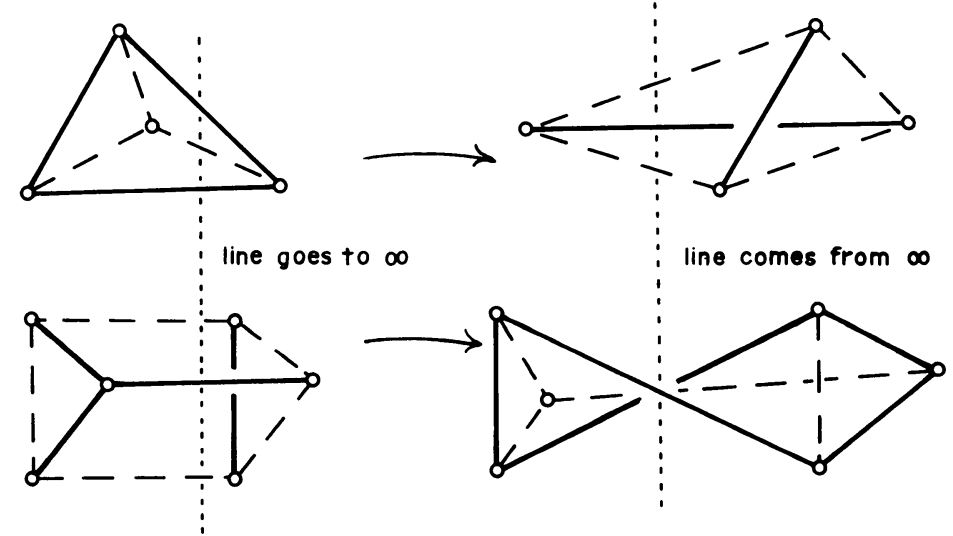

FIGURE 10 
We can now apply projective transformations to any of the frameworks in §§3-6 and obtain other results about the rigidity of some nonconvex polyhedra.

For example, what do the projectively transformed convex polyhedra look like? Some examples in Figure 11 illustrate the following possibilities.

(i) If the new plane at $\infty$ does not intersect the polyhedron then we obtain a new convex polyhedron (Figure 11A).

(ii) If the new plane at $\infty$ cuts off just one joint then we obtain a nonconvex spherical polyhedron with a concave dish and one sharp convex point (Figure 11B).

(iii) In general, when the new plane at $\infty$ cuts off more than one joint we obtain two concave dishes joined by a tangled mass of self-intersecting faces (Figure 11C).

If we inspect our original definitions then we find that the projective image of any strict polyhedral framework is a new strict polyhedral framework, on the same
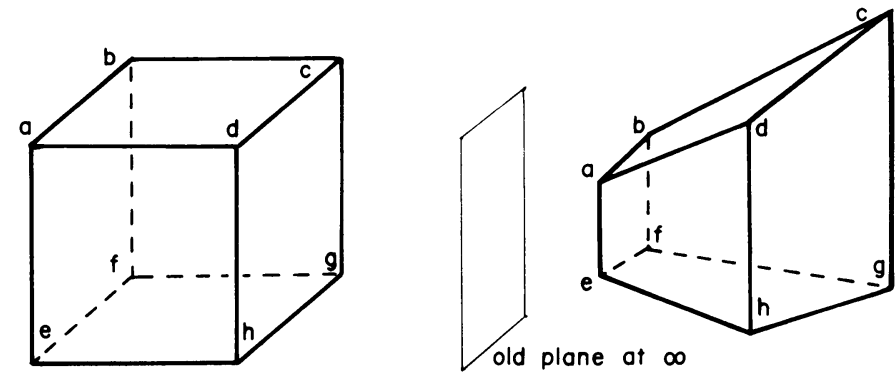

A

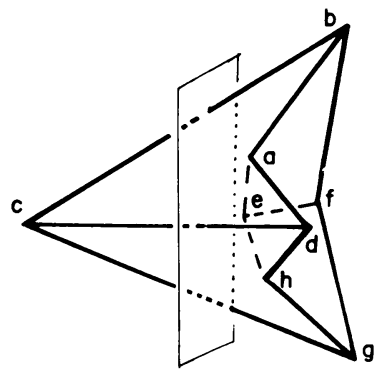

B

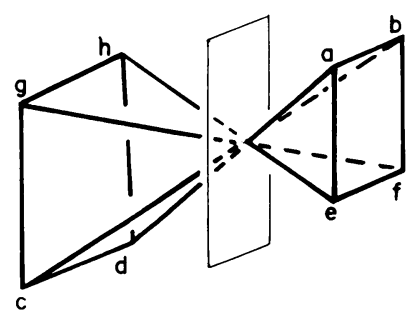

C

FIGURE 11

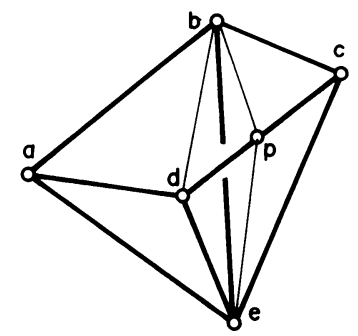

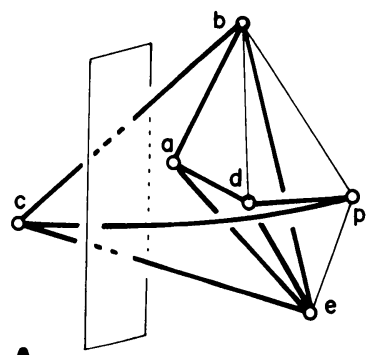

A

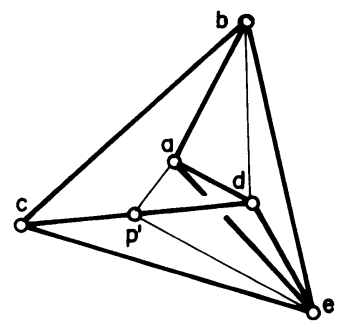

B

FIGURE 12 
abstract polyhedron. We can apply (or reapply) the modifications discussed in §§4-6 to these polyhedra-adding new joints along the "new natural edges" and in the "new natural faces". All the theorems generalize to give appropriate static-rigidity, independence, etc.

If an edge or face was cut by the new plane at infinity, the whole sense of "interior" has changed but the line and plane are not changed. Thus joints added "along" a natural edge now lie "outside" the polyhedron, creating strange flanges (Figure 12A). However, a careful examination of all the proofs in \$§4-6 support the observation that we never really used "inside" or "outside"-just the lines and planes, and a clear identification of added joints with edges or faces of the original polyhedron. Thus it is not important whether we add these new joints before or after projection-and we are certainly free to rework the faces after projection, whether they are convex or concave (Figure 12B).

REMARK. Second-order rigidity is not a projective invariant. Nevertheless the proof employed by Connelly is projectively invariant-provided that the faces are triangulated as convex polygons and then projected, or the faces are convex after projection, and are retriangulated then. Thus we get the second-order rigidity of some strange nonconvex polyhedra with flanges (Figure 13A). It would be interesting to know more about the orders of rigidity which occur when such nonconvex faces are directly retriangulated in their interiors after projection (Figure 13B).

8. Convex polytopes in $N$-space. So far we have concentrated on polyhedra in 3-space. Of course, in passing, we observed how a convex polygon in the plane could move, since our description of sign changes at a convex vertex can be viewed as a description of which interior angles increased or decreased as the convex polygon (a slice near the joint) moved in the plane. Proposition 2.5 gives a simple solution to the problem of making a convex polygon plane-statically rigid-simply triangulate the polygon.

What happens in 4-space? It turns out to be even easier to make 4-polytopes statically rigid in 4-space. The spirit of the proof is this. The 4-polytope is constructed so that a slice with a hyperplane (3-space) near the vertex is a convex polyhedron. This convex polyhedron is statically rigid in its 3-space and hence has no internal infinitesimal motions. However, any internal motion of the 4-polytope, involving edges at this vertex, would appear as an internal motion of such a general

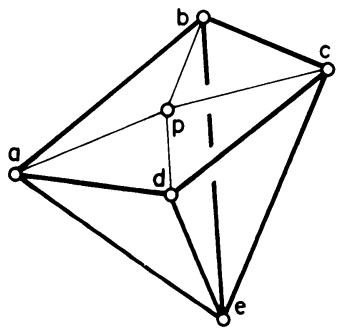

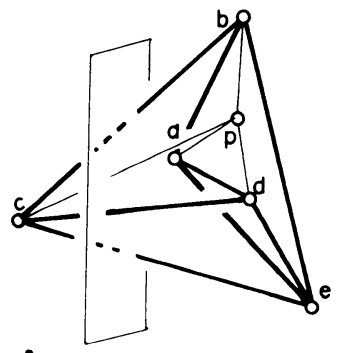

A

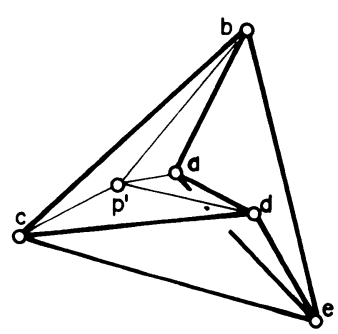

B

FIGURE 13 
slice, so we conclude that this vertex, and its adjacent edges, form an infinitesimally rigid block in 4-space. If this happens at enough vertices (e.g. all vertices of a convex 4-polytope) then we conclude that all of the 4-polytope is infinitesimally rigid.

This general flavour has been observed by others [21, footnote on p. 119]. However we have not located any direct presentation and it is unclear whether the basic objects are 4-polytopes composed of 4-space rigid cells, or 3-space rigid cells. Are we seeing an extension of Cauchy's result (which assumed 3-space rigid 2-faces) or Alexandrov's result (which assumed plane-rigid 2-faces)?

We will present the 4-dimensional analogue (actually analogues) of Alexandrov's results in some detail. Following our approach on 3-space, we begin with the generalization of Theorem 3.1, using only natural vertices. We then modify the frameworks to allow vertices on any natural edges (1-faces), natural planes (2-faces) or natural cells (3-faces), and to allow natural static replacements. This entire pattern extends to any higher dimension without any difficulty, and we state, without proof, such a result. However, we anticipate that the case in 4-space is sufficient to yield a different (higher?) perspective on the remarkable coincidence of triangulations and static rigidity which lives in 3-space.

We begin with our definition of an abstract oriented 4-polytope, and of the corresponding strict realizations.

DEFINITION 8.1. An abstract oriented 4-polytope is a set of vertices $V=\left\{a_{1}, \ldots, a_{v}\right\}$ and a set of oriented spherical polyhedra $\left\{P_{1}, \ldots, P_{n}\right\}$ (the cells) such that:

(i) the vertices of each cell $P_{i}$ lie in $V$,

(ii) each 2-face (a polygonal face of some $P_{i}$ ) appears in exactly two cells, and has opposite orientation (as a cycle of vertices) in the two cells,

(iii) for each edge $a_{i} a_{j}$ (adjacent vertices in a 2-face) there is a cycle of cells $P_{K_{1}}, P_{K_{2}}, \ldots, P_{K_{m}}(m \geqslant 3)$ such that two adjacent cells share a 2 -face containing the edge $a_{i} a_{j}$, and all polyhedra containing this edge appear in the cycle,

(iv) for each vertex $a_{i}$ there is a dual, abstract spherical polyhedron formed with dual vertices $\left\{P_{j} \mid P_{j}\right.$ is a cell containing $\left.a_{i}\right\}$ and dual faces - the cycles $P_{j_{1}} \cdots P_{j_{m}}$ for all edges $a_{i} a_{j}$ containing $a_{i}$.

REMARK. For readers who have never "seen" a 4-polytope, consider the 4-simplex formed of all 5-tetrahedra on the 5 vertices $a, b, c, d, e$ (Figure 14A). The 2-faces are all triangles on the 5 vertices, and the dual polyhedra at the vertices will also be tetrahedra. See [12] for examples of regular 4-polytopes, and [13] for a general discussion of convex 4-polytopes.

Definition 8.2. A strict 4-polytope is a realization of the vertices of an abstract oriented 4-polytope by points in 4-space such that:

(i) the vertices of each 3-cell span a single 3-dimensional subspace (hyperplane) and the cell forms a strict polyhedron in this subspace,

(ii) two cells sharing a common face lie in different 3-spaces.

DEFINITION 8.3. A strictly convex 4-polytope is a strict 4-polytope such that the hyperplane for each cell places all vertices not in the cell into a single open half-space.

It is a clear consequence of Definition 8.3 that each cell of a strictly convex 4-polytope is a strictly convex polyhedron. Less obvious, but more important for this 
work, is the fact that if we slice into the 4-polytope near a vertex $a_{0}$ with a hyperplane $H$, which if translated to $a_{0}$ would miss all the rest of the polytope, then the cross-section is a convex 3-polyhedron.

Why? Assume this hyperplane $H$ meets an edge $a_{0} a_{i}$ at the point $e_{i}$. It therefore meets a face containing $a_{i} a_{0} a_{j}$ in an edge $e_{i}, e_{j}$, and meets each polyhedral cell containing $a_{0}$ in a polygonal face $e_{k_{1}}, \ldots, e_{k_{m}}$. Since each 2-face of the 4-polytope was shared by two cells, each new edge $e_{i} e_{j}$ is shared by two of the new faces. Finally since the hyperplane for $P_{k}$ placed all other vertices (and edge points $e_{i}$ ) in a single open half-space, its intersection with $H$, the face plane of its polygon, place all other $e_{i}$ into a single half-space of $H$. This verifies the convexity of the section.

We want to construct our 4-polytopes as bar and joint frameworks. We offer a general definition, and then a restricted definition for convex 4-polytopes.

DEFINITION 8.4. A strict 4-polytopial bar and joint framework is a bar and joint framework built on a strict 4-polytope by

(i) placing a joint at each vertex of the polytope,

(ii) placing a bar on each edge of the 4-polytope,

(iii) placing some additional bars joining pairs of joints which lie on the same cell.

DEFINITION 8.5. A strictly convex 4-polytopial framework is a bar and joint framework built on a strictly convex 4-polytope by

(i) placing a joint at each vertex of the polytope,

(ii) placing a bar on each edge of the 4-polytope,

(iii) placing, on each 2-face of the polytope, a set of bars which triangulate the convex plane polygon.

We can give the 4-space analogue of Theorem 3.1.

THEOREM 8.1. A strictly convex 4-polytopial framework is infinitesimally rigid in 4-space.

Proof. The proof is based on two observations. If a convex 4-polytopial framework has an internal instantaneous motion $\left\langle\ldots, v_{i}, \ldots\right\rangle$, then there are 3 adjacent joints $a_{h} a_{0} a_{k}$ such that $\left(v_{h}-v_{k}\right) \cdot\left(a_{h}-a_{k}\right) \neq 0$. Secondly, an appropriate crosssection of this framework near $a_{0}$ will also have an internal motion.

We begin by establishing the first principle. Assume that we have an instantaneous motion of our framework $\left\langle\ldots, v_{i}, \ldots\right\rangle$. Further, assume that for each set of 3 adjacent joints $a_{h}, a_{0}, a_{k}$ we have the condition $\left(v_{h}-v_{k}\right) \cdot\left(a_{h}-a_{k}\right)=0$. We will prove that this instantaneous motion extends to a rigid motion of all space.

Take a joint $a_{0}$. Its 2-link consists of all bars $\{0, i\}$ and joints $a_{i}$ attached to this joint. Since every pair $a_{i} a_{j}$ lies on a set of 3 adjacent joints, we have, for all pairs, $\left(v_{i}-v_{j}\right) \cdot\left(a_{i}-a_{j}\right)=0$. In addition, the joints $a_{i}$ span 4-space (in an affine sense) so these velocities on the link extend to a rigid motion of 4-space $R_{0}$. If we go out a natural edge to an adjacent joint $a_{1}$, then we find that its 2-link is undergoing the rigid motion $R_{1}$. However, for each 2 -face of the polytope containing the edge $a_{0} a_{1}$ there is a triangle of bars $a_{0} a_{1} a_{j}$ and these $a_{j}$ belong to both links. We conclude that $R_{0}$ and $R_{1}$ must agree on all $a_{j}$, and $a_{0}, a_{1}$, a set of points spanning 4-space. Thus 
$R_{0}=R_{1}$. If we move on down a path of natural edges to any other joint we will find that all links, and therefore all joints are undergoing the rigid motion $R_{0}$, as required.

We now assume that the 4-polytope has an instantaneous motion with 3 adjacent joints $a_{h} a_{0} a_{k}$ satisfying $\left(v_{h}-v_{k}\right) \cdot\left(a_{h}-a_{k}\right) \neq 0$. We take a slice near $a_{0}$ with a hyperplane $H$, creating the point $e_{i}$ for each bar $a_{0} a_{i}$. Clearly in 4-space these $e_{i}$ have velocities $u_{i}$ from the bars, and

$$
\left(u_{i}-u_{j}\right) \cdot\left(e_{i}-e_{j}\right)=0 \text { iff }\left(v_{i}-v_{j}\right) \cdot\left(e_{i}-e_{j}\right)=0 .
$$

We now transfer these $u_{i}$ into the 3-space $H$. Take a unit normal $N$ (in 4-space) for this hyperplane, and define $w_{i}=u_{i}-\left(u_{i} \cdot N\right) N$. The $w_{i}$ are the projections, of $u_{i}$ into $H$, down the normal and, since $e_{k}, e_{j}$ are in $H$ we have

$$
\begin{aligned}
\left(w_{i}-w_{j}\right) \cdot\left(e_{i}-e_{j}\right) & =\left(u_{i}-u_{j}+\left(u_{i} \cdot N\right) N-\left(u_{j} \cdot N\right) N\right) \cdot\left(e_{i}-e_{j}\right) \\
& =\left(u_{i}-u_{j}\right) \cdot\left(e_{i}-e_{j}\right) .
\end{aligned}
$$

Now examine the cross-section as a convex polyhedron. For each face of this polyhedron we had a convex polyhedral framework in the 4-polytope. By Theorem 3.1 this framework is statically-hence infinitesimally-rigid in its 3-space, so for $e_{i}$, $e_{j}$ coming from this framework $\left(u_{i}-u_{j}\right) \cdot\left(e_{i}-e_{j}\right)=0$. We conclude that, even after projection, $\left(w_{i}-w_{j}\right) \cdot\left(e_{i}-e_{j}\right)=0$ for each pair of joints on a face and the $w_{i}$ form an allowed instantaneous motion for modified strictly convex polyhedral frameworks on the $e_{i}$ (with some $e_{i}$ on natural edges of the cross-section). However, we assumed that $\left(v_{h}-v_{k}\right) \cdot\left(a_{h}-a_{k}\right) \neq 0$ and therefore $\left(w_{h}-w_{k}\right) \cdot\left(e_{h}-e_{k}\right) \neq 0$ on the section. This contradicts Corollary 4.4 .

This completes the proof that all sets of 3 adjacent joints form a rigid triangle and hence, that the framework is infinitesimally rigid in 4-space.

REMARK 1. From the proof it is clear that we could assume much less than strict convexity. What we needed was a connected tree to all vertices, such that at all vertices which are not 1-valent, a slice forms a 3-space statically rigid polyhedron (e.g. anything satisfying theorems in §§4-7). In addition, we need to know that each cell has a subframework which is 3 -space statically rigid.

For example, if we build a simple strict 4-polytope (exactly 4 cells at each vertex) with cells which are 3 -space statically rigid, then all sections will be noncoplanar tetrahedra and the entire framework will be 4-space statically rigid. (It is a nice exercise to understand why the analogous claim for simple polyhedra (polyhedra in 3-space with 3 faces at each joint) is false.)

In the spirit of $\$ \S 4-6$ we can now proceed to add new joints along the edges, 2-faces and even 3-faces (cells) of a statically rigid 4-polytopial framework. We begin with the addition of joints along natural edges.

THEOREM 8.2. If a statically rigid strict polytopial framework is modified by

(i) the addition of new joints along the natural edges of the 4-polytope,

(ii) the replacement of each cell by a new subframework on all the joints of the cell which is 3-space statically rigid

then the modified framework is statically rigid in 4-space. 
Proof. We first add the new joints as 4-valent joints-with one bar to one end of the natural edges and one bar down each of 3 different faces at this edge-so that these 4 bars are not cospatial. This is always possible by the strict form of the 4-polytope. By Corollary 2.2, this creates a 4-space statically rigid framework.

We can now perform a static replacement on each cell. By Theorem 2.6, the modified framework is statically rigid in 4-space.

If the original strict 4-polytopial framework only had bars in the 2-faces, then Theorem 8.2 also takes a simpler form. We give the obvious case.

COROLLARY 8.3. If a strictly convex 4-polytopial framework is modified by

(i) the addition of new joints along natural edges,

(ii) the replacement of 2-faces by subframeworks which are plane-statically rigid then the modified framework is statically rigid in 4-space.

Proof. If we follow the proof of Theorem 8.2 we find that all bars lay in 2-faces, and static replacements in the 2 -faces will create the desired modified framework.

REMARK 1. If an edge has more than 3 adjacent faces (and cells) then the insertions described will actually add more than 4 bars for each new joint, and these will contribute to an overbracing of the structure. We will return to a discussion of this overbracing later.

REMARK 2. The occurrence of 4-polytopial frameworks, statically rigid in 4-space with large cells and all braces in their 2-faces, is in surprising contrast to events in 3-space. Imagine such a 4-dimensional "house". If you looked out at such a cell, your line of vision would normally meet a point in the interior of the cell, and you could see right through with no obstructions. The cell would be a large "window", with all the bars in the framing 2-faces. The entire house would be big windows, with bars and joints only in the frames, and the framework would still be statically rigid. In contrast, in 3-space a sphere can only have triangular windows if it is to be statically rigid!

We can now go further, and add joints on the natural 2-faces.

THEOREM 8.4. If a statically rigid strict 4-polytopial framework is modified by

(i) adding new joints along natural edges and on natural 2-faces,

(ii) replacing each 3-cell by a new subframework which is statically rigid in its own 3-space (on all the new joints associated with the cell), then the modified framework is statically rigid.

Proof. As before, we add the new joints along the natural edges as 4-valent joints.

For new joints along natural faces, we place a 4-valent joint with two bars (not collinear) in that face, and one bar, off this face, into each of the two cells adjacent to the face. This is now a statically rigid framework, and the static replacements on the 3-cells carry the static rigidity to the modified framework.

REMARK. Unlike some joints on natural edges, these new facial joints do not contribute to an overbracing, provided the rerlacement cells are isostatic. Thus their behaviour has analogies with the role of edge joints in 3-space. 
If we add facial joints, but keep all our bars in the 2-faces, then we have a new count of the infinitesimal degrees of freedom extending Theorem 6.1. We give a simple case.

COROLLARY 8.5. If a strictly convex 4-polytopial framework is modified by

(i) the addition of new joints on the natural edges,

(ii) the addition of $K$ new joints on the 2-faces,

(iii) the replacement of 2-faces by subframeworks which are plane-statically rigid, then the modified framework has exactly $2 K$ degrees of internal freedom, and these internal motions are generated by 2 vibrations normal to the face plane of each facial joint.

Proof. In the cell replacement of Theorem 8.4 we use a framework with 1 interior bar connecting each facial joint to a natural vertex of the cell. By the proof of Theorem 6.1 this cell framework is 3-space statically rigid, so the entire new modified framework is statically rigid, and thus equivalent to the original modified framework (with face replacements) plus 1 added bar per facial vertex and adjacent -a total of $2 K$ added bars.

If we remove these $2 K$ interior bars, then we introduce the $2 K$ vibrations described in the statement of the theorem. (There are 2 directions normal to each plane in 4-space.) Since we have introduced at most $2 K$ degrees of internal freedom, and we have described $2 K$ independent internal motions, we conclude that there are exactly $2 K$ degrees of internal freedom.

REMARK 1. It is not difficult to see that Corollary 8.5 could be used to extend Connelly's 2nd-order rigidity results to 4-polytopes:

If a strictly convex 4-polytope has all 2-faces arbitrarily triangulated, then the resulting framework is 2 nd-order rigid in 4-space.

REMARK 2. We can give results, analogous to Corollary 8.5, for inserting new joints inside the cells. When we replace the cells by 3 -space statically rigid frameworks the result will be 1 extra instantaneous vibration for each cellular joint (normal to its cell). Again suitable 2nd-order conclusions can be obtained.

In our discussion of 4-polytopes we have been careful not to talk about independence, or a count of stresses. In general, a convex 4-polytope with all 2-faces triangulated will already be overbraced, and thus stressed. We offer a few examples which are small enough to be isostatic in 4-space, and then a slightly larger example which is 1-overbraced.

The 4-simplex illustrated in Figure 14A has 5 joints and 10 bars. In an isostatic 4-space framework (more than 3 joints) we need $4|V|-10$ bars. Since this framework is convex unless flat, it is, in general, an isostatic framework.

In Figures 14B and D we illustrate a few more frameworks which have $4|V|-10$ bars, and can be built as convex 4-polytopes (hence isostatic).

However the example in Figure 14C, which can also be convex, has $4|V|-9$ bars. It will, in general, have one stress. A bit of additional computation confirms that it is, in general (including all strictly convex forms), a circuit - a framework in which removing any one bar will leave an isostatic framework. To a 4-space engineer this 
could be viewed as "optimally 1-overbraced", or as a structure which could be built as a statically rigid tensegrity framework.

Larger 4-polytopes will be inevitably overbraced. It would be very difficult to give general theorems to describe isostatic or independent frameworks built around 4-polytopes. For example, a triangulated tesseract (4-cube) will become a 2-overbraced framework. It is an interesting exercise to describe which subframeworks of this pattern are isostatic. The 3-sphere represents a happy coincidence among all closed manifolds of any dimension!

We close with a statement of the general theorems for convex $d$-polytopes. The definitions follow our established pattern, and will be omitted.

THEOREM 8.6. If a strictly convex d-polytope $(d \geqslant 3)$ is formed in $d$-space by

(i) the placement of a joint at each natural vertex,

(ii) the replacement of each 2-face by a subframework which triangulates the polygon, then the strictly convex $d$-polytopial framework is statically rigid in $d$-space.

THEOREM 8.7. If a strictly convex d-polytope is formed as a framework by

(i) the placement of a joint at each natural vertex,

(ii) the placement of joints along natural faces of $\operatorname{dim} \leqslant K \leqslant d-2$,

(iii) the replacement of all $(K+1)$-faces by subframeworks, on all joints associated with the face, which are statically rigid in their $(K+1)$-space,

then the modified framework is statically rigid in $d$-space.

9. Conclusions. We have managed extensive analyses of statically rigid and of independent frameworks based on convex spheres and their projections. We have also offered general results for the modification of "other" statically rigid polyhedral

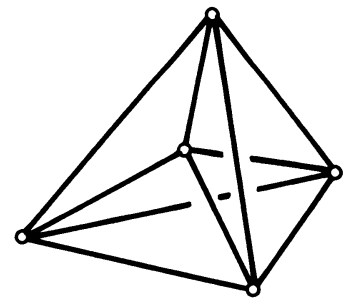

A

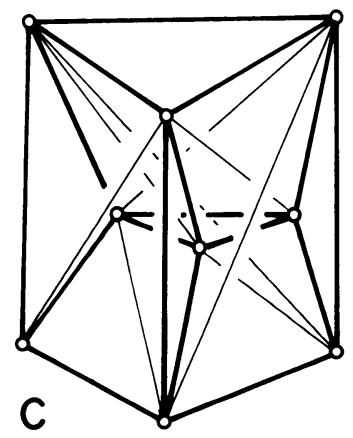

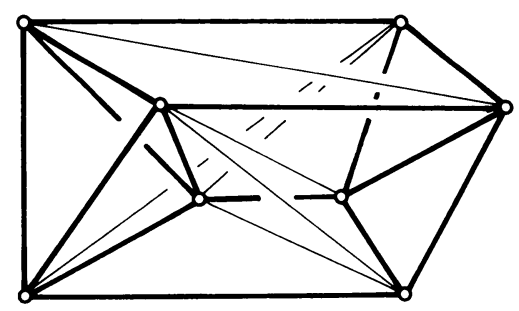

B

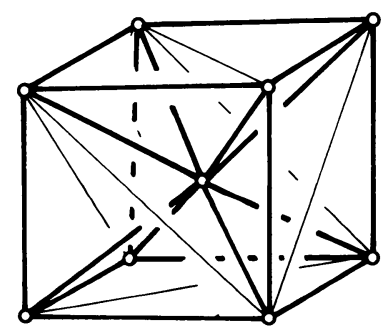

D

FIGURE 14 
frameworks. However, we have said very little about what these "other" polyhedral frameworks might be.

Very little is known about other classes of realizations of, say, a triangulated sphere, which will always be statically rigid. The general theory runs as follows. Given an abstract graph (vertices and edges) it can be realized in 3-space as a bar and joint framework. If it counts to be isostatic $(|E|=3|V|-6)$ then the static rigidity can be measured by the rank of the rigidity matrix [2, Theorem 4.1]. This, in turn, can be measured by whether certain determinants are zero [15, 22]. (Actually one determinant is needed, if we are careful $[22, \S 4]$.) Thus the realizations which are not statically rigid form an algebraic subspace of $\mathbf{R}^{3 V}$, and all of the rest of $\mathbf{R}^{3 V}$ consists of realizations which are statically rigid. If there is even one statically rigid realization, this guarantees that the statically rigid realizations form an open, dense subset of $\mathbf{R}^{3 V}$, and almost all realizations are statically rigid $[2,15]$.

Our ultimate task is not to refine our techniques for carving out open pieces of $\mathbf{R}^{3 \mathrm{~V}}$ (like the convex realizations of triangulated spheres) which are statically rigid. Rather the goal should be to describe, or confine that "small" set of geometrically bad realizations. Since static rigidity is projectively invariant, we should expect the description to lie within projective geometry [5, 11, 23].

We offer two simple examples of this goal. Consider the octahedral graph (Figure 15A). It is well known that a realization of this graph is statically rigid unless four alternate face planes concur in a single point (in projective space) (Figure 15B).

Our second example is dual to this - the graph of a cube with the faces triangulated (Figure 15C). We do not know the description for all bad realizations of this graph, but if the cube is realized so that each of its 6 faces is a plane, then the framework fails to be statically rigid just when four alternate joints $\left(a, d, b^{\prime}, c^{\prime}\right)$ are coplanar (Figure 15D). In particular, all realizations without self-intersection are statically rigid. This last observation is a special case of a general pattern: If any "prism" is realized in 3-space with triangulated plane faces and no self-intersection, then it is statically rigid [28].

The general problem of characterizing, geometrically, the "bad" realizations, even of a triangulated sphere, remains an important unsolved problem.

In a companion paper to this, we will investigate the infinitesimal rigidity of panel polyhedra-polyhedra constructed with infinitesimally rigid panels in place of some, or all of the faces. This paper will build on the present static results, but the analysis will be based on infinitesimal motions and on a special correspondence between motions and stresses presented in [11]. In this context a number of other extensions to nonconvex polyhedra are available [27].

In closing, we mention two other related areas of research which extend the methods developed here.

We know, in general, no particular statically rigid realization of a typical triangulation of the torus. However, the techniques of static insertion and static replacement have been employed to obtain the result that, for each triangulation of the torus, almost all realizations are statically rigid. We have combined the same techniques with results from graph theory, to obtain the static rigidity of almost all realizations 

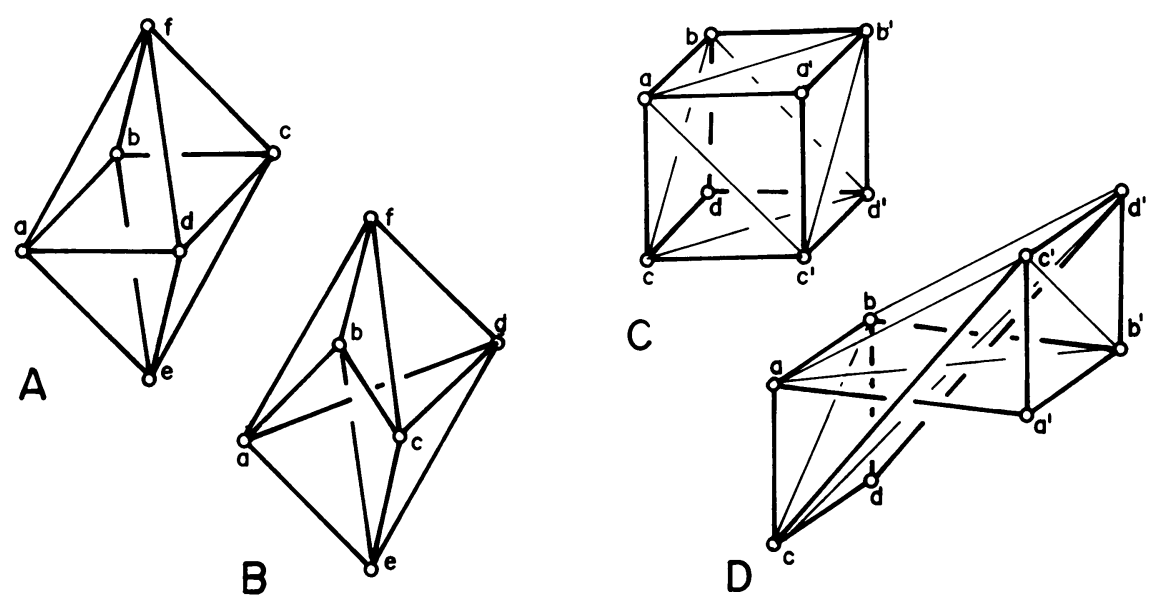

\section{FIGURE 15}

of special graphs obtained by adding one or several shafts to a triangulated sphere, and then deleting certain designated edges of the original triangulation.

Finally the repeated replacements of one plane-statically rigid face by another such framework has brought home the point that we can really let all details of this plane framework face away, and work with a "plane-rigid sheet" on the designated joints. From this (and other) experiences we have developed a general static theory of such plane-rigid sheets in 3-space, and obtained some surprising results. We will just tantalize the reader with one crucial theorem:

A space structure formed of plane-rigid sheets joined in pairs along folds (line segments common to two sheets) is the projective dual of a bar and joint framework, and this duality preserves all static (and infinitesimal kinematic) properties, including static rigidity [28].

\section{REFERENCES}

1. A. D. Alexandrov, Konvex polyeder, German transl., Akademie-Verlag, Berlin, 1958.

2. L. Asimow and B. Roth, Rigidity of graphs, Trans. Amer. Math. Soc. 245 (1978), 279-289.

3. __ Rigidity of graphs. II, J. Math. Anal. Appl. 68 (1979), 171-190.

4. J. Baracs, Rigidity of articulated panel structures, Bull. Internat. Assoc. for Shell and Spacial Structures 59, Vol. XVI-3, Madrid, 1975.

5. __ Introduction, Structural Topology 1 (1979), 8-12.

6. R. Bricard, Memoire sur la theorie de l'octaedre articule, J. Math (Liouville) (5) 3 (1897), 113-148. $27-44$.

7. E. D. Bolker and B. Roth, When is a bipartite graph a rigid framework? Pacific J. Math. 90 (1981),

8. A. Cauchy, Deuxieme memoire sur les polygons et les polyedres, J. Ecole Polytechnique XVIe Cahier (1831), 87-98.

9. R. Connelly, The rigidity of polyhedral surfaces, Math. Mag. 52 (1979), 275-283.

10. The rigidity of certain cabled frameworks and the second order rigidity of arbitrarily triangulated convex surfaces, Adv. in Math. 37 (1980), 272-298.

11. H. Crapo and W. Whiteley, Statics of frameworks and motions of panel structures: a projective geometric introduction, Structural Topology 6 (1982), 42-82.

12. H. S. M. Coxeter, Regular polytopes, Dover, New York, 1973.

13. B. Grünbaum, Convex polytopes, Wiley, New York, 1968.

14. , Lectures in lost mathematics, University of Washington, Seattle, Washington, 1976, preprint. 
15. H. Gluck, Almost all simply connected closed surfaces are rigid, Geometric Topology, Lectures Notes in Math., vol. 438, Springer-Verlag, Berlin and New York, 1975, pp. 225-239.

16. L. Henneberg, Die Graphische Statik der Starren Systeme, Liepzig, 1911; Johnson reprint, 1968.

17. N. H. Kuiper, Sphères polyedriques flexible dans $E^{3}$, d'après Robert Connelly, Sem. Bourbaki 514 (1978), 514-101-514-12.

18. J. C. Maxwell, On reciprocal figures and diagrams of forces, Philos. Mag. Ser. (4) 27 (1864), 250-261.

19. B. Roth, Rigid and flexible frameworks, Amer. Math. Monthly 88 (1981), 6-20.

20. B. Roth and W. Whiteley, Rigidity of tensegrity frameorks, Trans. Amer. Math. Soc. 256 (1981), 419-446.

21. J. J. Stoker, Geometric problems concerning polyhedra in the large, Comm. Pure Appl. Math. 21 (1968), 119-168.

22. N. White and W. Whiteley, The algebraic geometry of stresses in frameworks, J. Algebraic Discrete Methods 4 (1983), 481-511.

23. W. Whiteley, Motions, stresses and projected polyhedra, Structural Topology 7 (1983), 13-38.

24. __ Realizability of polyhedra, Structural Topology 1 (1979), 46-58.

25. Introduction to structural geometry. I. Infinitesimal motions and infinitesimal rigidity, Champlain Reg. Coll., St. Lambert Quebec, preprint 1977.

26.

Lambert, Quebec, preprint 1978.

27. __ Infinitesimally rigid polyhedra. II. Articulated panels (to appear).

28.

Department of Mathematics, Champlain Regional College, St. Lambert, Quebec J4P 3P2, CANADA (Current address)

Topologie Structurale, Ecole d'Architecture, Universite de Montreal, Montreal, Quebec H3C 3J7, Canada 